\title{
Automorphism Groups of Symmetric and Pseudo-real Riemann Surfaces
}

\author{
Ewa Tyszkowska®
}

\begin{abstract}
The category of smooth, irreducible, projective, complex algebraic curves is equivalent to the category of compact Riemann surfaces. We study automorphism groups of Riemann surfaces which are equivalent to complex algebraic curves with real moduli. A complex algebraic curve $C$ has real moduli when the corresponding surface $X_{C}$ admits an anti-conformal automorphism. If no such an automorphism is an involution (symmetry), then the surface $X_{C}$ is called pseudo-real and the curve $C$ is isomorphic to its conjugate, but is not definable over reals. Otherwise, the surface $X_{C}$ is called symmetric and the curve $C$ is real.

Mathematics Subject Classification. Primary 30F99; Secondary 14H37, $20 \mathrm{~F}$.

Keywords. Riemann surface, Symmetry of a Riemann surface, Asymmetric Riemann surface, Pseudo-symmetric Riemann surface, Fuchsian groups, NEC groups .
\end{abstract}

\section{Introduction}

It is known that the moduli space $\mathcal{M}_{g}$ of complex algebraic curves of genus $g$ is a quasi-projective variety which can be defined in $\mathbb{P}^{n}(\mathbb{C})$ by polynomials with rational coefficients. There is an anti-holomorphic involution $\iota: \mathcal{M}_{g} \rightarrow$ $\mathcal{M}_{g}$ which maps the class of a complex curve to its conjugate. The fixed points of such a mapping are called complex algebraic curves with real moduli. The defining equations of symmetric Riemann surfaces are over reals, and therefore, they are fixed by $\iota$. Earle [7] proved that not every curve with real moduli is a real curve. Seppälä [22] showed that the non-real complex algebraic curves with real moduli are coverings of real algebraic curves. They are called pseudo-real or asymmetric curves.

The existence of pseudo-real surfaces of every genus $g \geq 2$ was proved in [5]. A characterization of automorphism groups of such surfaces and the minimal genus for the cyclic case was given in [2]. The topological types of actions of automorphism groups of pseudo-real surfaces of low genera were described 
in [4]. The pseudo-real surfaces with cyclic automorphism groups were studied in [9]. The hyperelliptic pseudo-real Riemann surfaces were considered in $[3,19]$ and $[6]$. The equations for non-hyperelliptic surfaces were found in [10] and [11]. The present paper can be seen as a continuation of papers [12-14,24], where asymmetric $p$-hyperelliptic and $(q, n)$-gonal surfaces, with cyclic automorphism group, were studied.

Let $\operatorname{Aut}^{ \pm}(X)$ be the group of all conformal and anti-conformal automorphisms of a Riemann surface $X$ of genus $g \geq 2$, and let $\operatorname{Aut}^{+}(X)$ be the subgroup of all conformal automorphisms. We say that a finite group $H$ acts on the surface $X$, if it is isomorphic to a subgroup of $\operatorname{Aut}^{ \pm}(X)$. By $(X, Y)_{g}^{H}$, we denote a pair of Riemann surfaces $X$ and $Y$ of the same genus $g \geq 2$ which have automorphism groups $H_{X} \subseteq \operatorname{Aut}^{ \pm}(X)$ and $H_{Y} \subseteq \operatorname{Aut}^{ \pm}(Y)$, such that $H_{Y} \simeq H \simeq H_{X}$. In the paper, we deal with the so-called (a.s.)-pairs $(X, Y)_{g}^{H}$ for which $X$ and $Y$ are pseudo-real and symmetric surfaces, respectively.

The second chapter of the paper presents the most important facts from theory of non-euclidean crystallograpfic groups, called briefly NEC groups. They are discrete and co-compact subgroups of the group of isometries of the hyperbolic plane $\mathcal{H}$. Fuchsian groups are defined as discrete subgroups of conformal automorphisms of $\mathcal{H}$ and they are not always co-compact. However, in this paper, we will assume that they are co-compact and we will treat them as NEC groups. An NEC group not being Fuchsian group will be called a proper NEC group.

According to the Riemann uniformization theorem, a compact Riemann surface $X$ of genus $g \geq 2$ can be represented as the orbit space $\mathcal{H} / \Gamma$ for some torsion-free Fuchsian group $\Gamma$. Moreover, an automorphism group $H \subseteq$ $\operatorname{Aut}^{ \pm}(X)$ of such a surface can be represented as the quotient group $\Lambda / \Gamma$ for a proper NEC group or a Fuchsian group $\Lambda$ according to whether $H$ contains anti-conformal automorphisms or not. An action of $H$ on $X$ corresponds to a short exact sequence of homomorphisms $1 \rightarrow \Gamma \rightarrow \Lambda \stackrel{\theta}{\rightarrow} H \rightarrow 1$ and is denoted by $(\Lambda, \theta, H)$.

Let $H$ be a finite group acting on a closed Riemann surface of genus $g \geq 2$. Theorem 3.2 of chapter 3 provides a sufficient condition on $H$ to construct an action of $H^{\prime}=H \rtimes \mathbb{Z}_{2}$ on another closed Riemann surface $X^{\prime}$ of the same genus as $X$, such that $X / H$ and $X^{\prime} / H^{\prime}$ are topologically equivalent orbifolds and $\mathbb{Z}_{2}$ component is generated by a symmetry. This condition is provided by the existence of an automorphism $\varphi \in \operatorname{Aut}(H)$ of order 2 with certain properties on a set of (geometric) generators of $H$. It was solved by Singerman for a $(k, l, m)$-group $H$ generated by two elements $h_{1}$ and $h_{2}$ of orders $k$ and $l$, respectively, whose product has order $m$. He proved in [21] that if such a group has an automorphism $\varphi \in \operatorname{Aut}(H)$ induced by $\varphi\left(h_{1}\right)=h_{1}^{-1}, \varphi\left(h_{2}\right)=h_{2}^{-1}$ or $\varphi\left(h_{1}\right)=h_{2}^{-1}, \varphi\left(h_{2}\right)=h_{1}^{-1}$, then an action of $H$ on a Riemann surface $X$ can be extended to an action of the semidirect product $H \rtimes\langle\rho\rangle$ for a symmetry $\rho$, such that $\rho h_{i} \rho=\varphi\left(h_{i}\right)$ for $i=1,2$. In this paper, we prove that for any $(k, l, m)$-group $H$ and $n \in \mathbb{N}$, there is a symmetric Riemann surface of genus $g_{n}$ with an automorphism group $H \rtimes D_{2 n}$, where $g_{n}=1+2 n|H|$ or $g_{n}=1+2 n|H|\left(1-\frac{1}{k}\right)$ according to whether $\varphi\left(h_{1}\right)=h_{1}^{-1}$ 
and $\varphi\left(h_{2}\right)=h_{2}^{-1}$ or $\varphi\left(h_{1}\right)=h_{2}^{-1}$ and $\varphi\left(h_{2}\right)=h_{1}^{-1}$, respectively. This result is a particular case of Theorem 3.4 which claims that if a finite group $H$ has a minimal generating set $A=\left\{h_{1}, \ldots, h_{n}\right\}$ and there is an automorphism $\varphi \in \operatorname{Aut}(H)$ of order 2, such that for each $h_{i} \in A, \varphi\left(h_{i}\right) \in\left\langle h_{j}\right\rangle$ for some $h_{j} \in A$, then for any even $q \in \mathbb{N}$, there exists an extension of $H$ by a dihedral group $D_{q}=\langle v, \rho\rangle$ which acts on a symmetric Riemann surface in such a way that $\rho$ is a symmetry and $v$ is a conformal involution of the surface.

In chapter 4, we study finite group actions on asymmetric Riemann surfaces. Theorem 4.2 determines conditions on generating vectors of the full automorphism group of such a surface. We prove that if $A$ is a generating set of a finite group $H$ and $\mu_{A^{\prime}}=\operatorname{card}(A)-\sum_{h \in A^{\prime}} \frac{1}{\sharp(h)}$ for a subset $A^{\prime} \subseteq A$, then for any $k \in \mathbb{N}$ and $\varphi \in \operatorname{Aut}(H)$, such that $4 k \equiv 0(\sharp(\varphi))$, there is an asymmetric Riemann surface $X$ of genus $g=1+4 k|H| \mu_{A^{\prime}}$ for which $\operatorname{Aut}^{ \pm}(X)$ is isomorphic to the semidirect product $H_{\varphi, 4 k}=H \rtimes\left\langle\delta: \delta^{4 k}\right\rangle$ with respect to the action $\delta h \delta^{-1}=\varphi(h)$ for $h \in H$. If $A^{\prime}=A$ and $\varphi^{2}=\mathrm{id}_{H}$, then for any $k \in \mathbb{N}$, there is an (a.s.)-pair $(X, Y)_{g}^{H_{\varphi, 4 k}}$ with $g=1+4 k|H| \mu_{A}$.

The last chapter is devoted to actions without fixed points. We find the conditions on a generating vector of finite group $H$ for which there exists an (a.s)-pair $(X, Y)_{g}^{H}$, such that $Y / H$ is a hyperelliptic Klein surface. Finally, in Theorem 5.5, we prove that if $p=\frac{g-1}{n}\left(\frac{n}{2}-1\right)$ for $n, g \in \mathbb{N}$, such that $g \geq 3$ is odd and $n$ is an even divisor of $g-1$, then there exists a symmetric $p$ hyperelliptic Riemann surface of genus $g$ being $n$-sheeted unbranched covering of a hyperelliptic Klein surface of algebraic genus $\frac{2(g-1)}{n}+1$. In particular, for any odd $g \geq 3$, there exists a symmetric hyperelliptic Riemann surface of genus $g$ with two commuting symmetries.

\section{Preliminaries}

\subsection{Non-Euclidean Crystallographic Groups}

We shall use the combinatorial approach, based on theory of non-Euclidean crystallographic groups (in short NEC groups) which are discrete and cocompact subgroups of the group of isometries of the hyperbolic plane $\mathcal{H}$, including those which reverse orientation. If such a subgroup contains only orientation preserving isometries, then it is called a Fuchsian group. Macbeath and Wilkie [15], [25] associated with every NEC group $\Lambda$ a signature $\sigma(\Lambda)$, which determines its algebraic structure. It has the form

$$
\sigma(\Lambda)=\left(h ; \pm ;\left[m_{1}, \ldots, m_{t}\right] ;\left\{C_{1}, \ldots, C_{u},(-), . . v,(-)\right\}\right) .
$$

The orbit space $\mathcal{H} / \Lambda$ is a surface of topological genus $h$, having $u+v$ boundary components, and being orientable or not according to the sign being + or -. The numbers $m_{i} \geq 2$, called the proper periods, represent the branched indices over interior points of $\mathcal{H} / \Lambda$ in the natural projection $\mathcal{H} \rightarrow \mathcal{H} / \Lambda$. The non-empty brackets $C_{i}=\left(n_{i 1}, \ldots, n_{i s_{i}}\right) i=1, \ldots, u$ and the empty ones $(-) . . .(-)$ with $s_{i}=0$ for $i=u+1, \ldots, u+v$ are the period cycles. The numbers $n_{i j} \geq 2$, called the link periods, represent branched indices over the $i$ th hole of the surface. 
The group with the signature (1) has the presentation given by the following generators and relations:

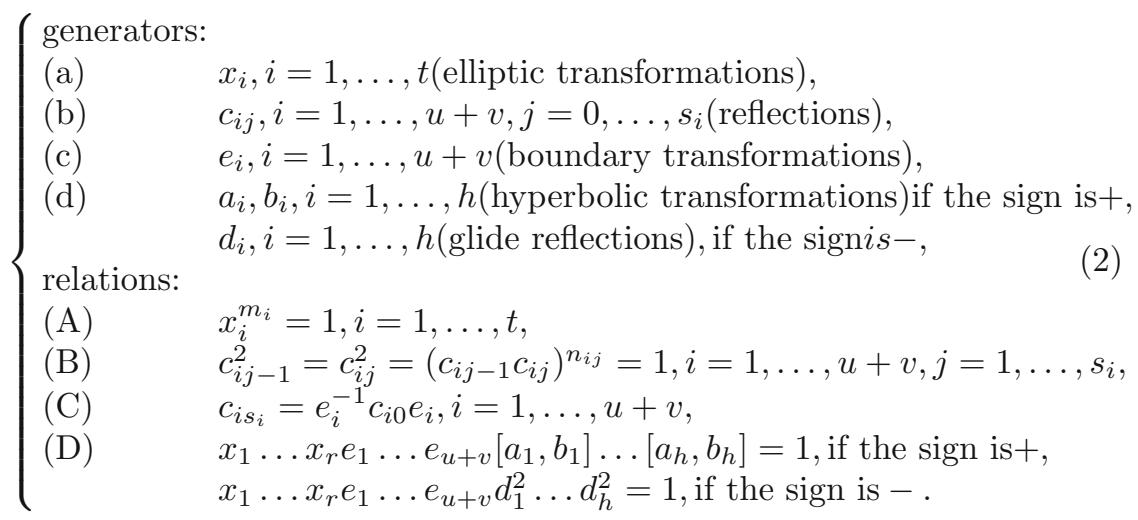

Any generators of an NEC group satisfying the above relations are called canonical generators.

The hyperbolic area $\mu(\Lambda)$ of any fundamental region of an NEC group $\Lambda$ with the signature (1) is given by

$$
2 \pi\left(\varepsilon h+u+v-2+\sum_{i=1}^{t}\left(1-\frac{1}{m_{i}}\right)+\frac{1}{2} \sum_{i=1}^{u} \sum_{j=1}^{s_{i}}\left(1-\frac{1}{n_{i j}}\right)\right),
$$

where $\varepsilon=2$ if the sign is + and $\varepsilon=1$ otherwise.

An abstract group with the presentation (2) can be realized as an NEC group if and only if (3) is positive.

If $\Gamma$ is a finite index subgroup of an NEC group $\Lambda$, then it is an NEC group itself and there is a Hurwitz-Riemann formula, which says that

$$
[\Lambda: \Gamma]=\frac{\mu(\Gamma)}{\mu(\Lambda)} .
$$

A Fuchsian group can be regarded as an NEC group with the signature $\left(h ;+;\left[m_{1}, \ldots, m_{t}\right] ;\{-\}\right)$, usually shortened to $\left(h ; m_{1}, \ldots, m_{t}\right)$.

An NEC group $\Lambda$ with the signature (1) has the so-called canonical Fuchsian subgroup $\Lambda^{+}$consisting of all conformal automorphisms in $\Lambda$. Singerman [18] proved that $\Lambda^{+}$has the signature

$$
\left(\varepsilon h+u+v-1 ; m_{1}, m_{1}, \ldots, m_{t}, m_{t}, n_{11}, \ldots, n_{1 s_{1}}, \ldots, n_{u 1}, \ldots, n_{u s_{u}}\right) .
$$

\subsection{Riemann Surfaces}

Any compact Riemann surface $X$ of genus $h>1$ is uniformized by a torsionfree Fuchsian group $\Gamma$ with the signature $(h ;-)$ called a surface Fuchsian group. An automorphism group $G$ of $X$ is isomorphic to a quotient group $\Lambda / \Gamma$ for some NEC group $\Lambda$ normalizing $\Gamma$. If there does not exist another NEC group containing $\Lambda$ properly, then $\Lambda$ is called a maximal $N E C$ group and $G=\Lambda / \Gamma$ is the full automorphism group of $X$. The detailed exposition of maximality can be found in [8]. 
A signature $\sigma$ is called maximal, if for every NEC group $\Lambda^{\prime}$ with a signature $\sigma^{\prime}$ containing an NEC group $\Lambda$ with the signature $\sigma$ and having the same Teichmüller dimension, the equality $\Lambda=\Lambda^{\prime}$ holds. For any maximal signature $\sigma$, there exists a maximal NEC group with the signature $\sigma$.

In the paper, we will use Macbeath's formula [16] on the total number of fixed points of a conformal automorphism of a Riemann surface.

Theorem 2.1. Let $H=\Lambda / \Gamma \subset \operatorname{Aut}^{+}(X)$ be an automorphism group of a Riemann surface $X=\mathcal{H} / \Gamma$. If $x_{1}, \ldots, x_{t}$ are canonical elliptic generators of $\Lambda$ with orders $m_{1}, \ldots, m_{t}$, respectively, and $\theta: \Lambda \rightarrow H$ is an epimorphism with kernel $\Gamma$, then the number $f(h)$ of points fixed by an automorphism $h \in H$ is given by the formula

$$
f(h)=\left|N_{H}(\langle h\rangle)\right| \sum_{i} \frac{1}{m_{i}},
$$

where $N_{H}(\langle h\rangle)$ is the normalizer of $\langle h\rangle$ in $H$ and the sum is taken over indices $i$ for which $h$ is conjugate to a power of $\theta\left(x_{i}\right)$.

\subsection{Klein Surfaces}

A Klein surface is a compact topological surface equipped with a dianalytic structure. A Riemann surface can be seen as an oriented Klein surface without boundary. For a given Klein surface $X$, Alling and Greenleaf [1] constructed certain double cover $X^{+}$being a Riemann surface. The algebraic genus of $X$ is defined as the genus of $X^{+}$, and for a surface of topological genus $g$ having $k$ boundary components, it is equal to $d=\alpha g+k-1$, where $\alpha=2$ if $X$ is orientable and $\alpha=1$ otherwise. Preston [17] proved that any compact Klein surface $X$ of algebraic genus $d \geq 2$ can be represented as $\mathcal{H} / \Gamma$ for the so-called surface $N E C$ group $\Gamma$. If $X$ has topological genus $g$ and $k$ boundary components, then $\sigma(\Gamma)=(g ; \pm ;[-] ;\{(-), . k .,(-)\})$. A finite group $G$ is a group of automorphisms of $X=\mathcal{H} / \Gamma$ if and only if $G \simeq \Lambda / \Gamma$ for some NEC group $\Lambda$ normalizing $\Gamma$.

It is said that a Klein surface $X$ is $p$-hyperellitic, if it has a an involution $\delta$ for which the orbit space $X /\langle\rho\rangle$ has algebraic genus $p$. The automorphism $\delta$ is called a $p$-hyperelliptic involution. If $p=0$, then the surface $X$ is called hyperelliptic.

\section{On Symmetric Generating Vectors}

A finite group $H$ acts on a Riemann surface $X$ of genus $g \geq 2$ if and only if there is a short exact sequence of homomorphisms

$$
1 \rightarrow \Gamma \rightarrow \Lambda \stackrel{\theta}{\rightarrow} H \rightarrow 1
$$

where $\Lambda$ is an NEC group and $\Gamma$ is a surface Fuchsian group isomorphic to the fundamental group of $X$. The action corresponding to this sequence is denoted by $(\Lambda, \theta, H)$. We say that $H$ acts with the signature $\sigma(\Lambda)$ and that $\theta$ is a smooth epimorphism. 
Two actions $(\Lambda, \theta, H)$ and $\left(\Lambda^{\prime}, \theta^{\prime}, H^{\prime}\right)$ on surfaces of the same genus $g$ are topologically equivalent if $\varphi \theta=\theta^{\prime} \psi$ for some isomorphisms $\varphi: H \rightarrow H^{\prime}$ and $\psi: \Lambda \rightarrow \Lambda^{\prime}$. An action $(\Lambda, \theta, H)$ is called full if it is topologically equivalent to an action of the full automorphism group of at least one of Riemann surfaces of genus $g$.

In this chapter, we will consider actions of a finite group $H$ with a signature

$$
\left(\gamma ; \pm ;\left[m_{1}, \ldots, m_{r}\right] ;\{-\}\right),
$$

where the sign is + for $\gamma=0$ and it is - otherwise. Let $\sharp(h)$ denote the order of an element $h \in H$. Then, we have the following.

Lemma 3.1. A finite group $H$ acts on a Riemann surface $X$ with the signature (6), if and only if it has a generating set $\left\{b_{1}, \ldots, b_{r}, h_{1}, \ldots, h_{\gamma}\right\}$, such that

$$
\prod_{i=1}^{r} b_{i} \prod_{j=1}^{\gamma} h_{j}^{2}=1 \text { and } \gamma-2+\sum_{i=1}^{r}\left(1-\frac{1}{\sharp\left(b_{i}\right)}\right)>0
$$

and no composition of generators containing an odd number of elements $h_{i}$ is trivial. The surface $X$ has genus

$$
g=1+\frac{|H|}{2}\left(\gamma-2+\sum_{i=1}^{r}\left(1-\frac{1}{\sharp\left(b_{i}\right)}\right)\right) .
$$

Proof. Suppose that there is an action $(\Lambda, \theta, H)$ for which $\Lambda$ has the signature (6). Then, $\Lambda$ is generated by glide reflections $d_{1}, \ldots, d_{\gamma}$ and elliptic elements $x_{i}, \ldots, x_{r}$ which satisfy the relations

$$
x_{i}^{m_{i}}=1 \quad \text { for } 1 \leq i \leq r \text { and } x_{1} \ldots x_{r} d_{1}^{2} \ldots d_{\gamma}^{2}=1 .
$$

Since $\Gamma=\operatorname{ker} \theta$ is a surface Fuchsian group, it follows that $H$ is generated by conformal automorphisms $b_{i}=\theta\left(x_{i}\right)$ of orders $m_{i}$ and anti-conformal automorphisms $h_{i}=\theta\left(d_{i}\right)$ which satisfy the relation $\prod_{i=1}^{r} b_{i} \prod_{j=1}^{\gamma} h_{j}^{2}=1$. Any product of these generators containing an odd number of elements $h_{i}$ is nontrivial, because otherwise $\Gamma=\operatorname{ker} \theta$ would have an anti-conformal automorphism. In particular, all elements $h_{i}$ have even orders. The hyperbolic area (3) of a fundamental region of $\Lambda$ is positive what implies that $\gamma-2+\sum_{i=1}^{r}\left(1-\frac{1}{\sharp\left(b_{i}\right)}\right)>0$.

Conversely, if a finite group $H$ has a generating set

$$
\left\{b_{1}, \ldots, b_{r}, h_{1}, \ldots, h_{\gamma}\right\}
$$

satisfying the conditions ( 7$)$ and any composition of these elements containing an odd number of $h_{i}$ is nontrivial, then there is an action $(\Lambda, \theta, H)$ on the Riemann surface $X=\mathcal{H} / \operatorname{ker} \theta$, where $\Lambda$ is an NEC group with the signature (6), such that $m_{i}=\sharp\left(b_{i}\right)$ for $1 \leq i \leq r$, and $\theta: \Lambda \rightarrow H$ is an epimorphism induced by

$$
\theta\left(d_{i}\right)=h_{i} \text { and } \theta\left(x_{j}\right)=b_{j} \quad \text { for } 1 \leq i \leq \gamma \text { and } 1 \leq j \leq r .
$$

By the Hurwitz-Riemann formula, the surface $X$ has genus (8). 
An ordered sequence $T_{r, \gamma}=\left[b_{1}, \ldots, b_{r}, h_{1}, \ldots h_{\gamma}\right]$ of generators of a finite group $H$ which satisfies the conditions of Lemma 3.1 is called a generating vector of $H$. We say that this vector is essential or unessential according to whether $\gamma>0$ or $\gamma=0$, respectively. In the first case $H$ has anti-conformal automorphism, and in the second case, it has only conformal automorphisms. We will also say that the vector $T_{r, \gamma}$ is exceptional, if the group $H$ has not a symmetry, by which we mean an anti-conformal automorphism of order 2 . Clearly, each unessential vector $T_{r, \gamma}$ is exceptional. In order to an essential vector $T_{r, \gamma}$ was exceptional, no composition of its elements containing an odd number of $h_{i}$ can have order 2 , because otherwise $H$ would have a symmetry. In particular, no $h_{i}$ can be an involution and so $\sharp\left(h_{i}\right) \equiv 0$ (4) for $1 \leq i \leq \gamma$.

Throughout the paper, we use the following notation. The symbol $m_{(n)}$ denotes $m \bmod n$ for $m, n \in \mathbb{N}$. If $\varphi$ is an automorphism of a finite group $H$ and $q \in \mathbb{N}$ is an integer, such that $q_{(\sharp(\varphi))}=0$, then $H_{\varphi, q}$ denotes the semidirect product of $H$ by a cyclic group $\mathbb{Z}_{q}=\langle\delta\rangle$ with respect to the action $\delta h \delta^{-1}=\varphi(h)$ for $h \in H$.

In a particular case, when $\varphi^{2}=\mathrm{id}_{\mathrm{H}}$ and $\varphi$ fixes a central element $z \in Z(H)$ of even order, then there is a normal subgroup $\left\langle z^{-1} \delta^{2}\right\rangle$ in $H_{\varphi, q}$ with $q=2 \sharp(z)$ and the quotient group $H_{\varphi, q} /\left\langle z^{-1} \delta^{2}\right\rangle$ will be denoted by $H_{\varphi / z}$.

Theorem 3.2. If a finite group $H$ has a generating vector $T_{r, \gamma}=\left[b_{1}, \ldots, b_{r}\right.$, $\left.h_{1}, \ldots, h_{\gamma}\right\rfloor$ and there is an integer $t$ in the range $1 \leq t \leq\left\lfloor\frac{r}{2}\right\rfloor$, such that

$$
\sharp\left(b_{i}\right)=\sharp\left(b_{2 t-i+1}\right) \quad \text { for } 1 \leq i \leq t
$$

and the assignment

$$
\begin{aligned}
b_{i} & \rightarrow b_{2 t-i+1}^{-1}, b_{t+i} \rightarrow b_{t-i+1}^{-1} \quad 1 \leq i \leq t \\
b_{2 t+i} & \rightarrow\left(\prod_{j=1}^{i-1} b_{2 t+j}\right) b_{2 t+i}^{-1}\left(\prod_{j=1}^{i-1} b_{2 t+j}\right)^{-1} 1 \leq i \leq r-2 t \\
h_{j} & \left.\rightarrow\left(\prod_{i=1}^{r-2 t} b_{2 t+i}\right)\left(\prod_{i=1}^{j-1} h_{i}^{2}\right) h_{j}^{-1}\left(\prod_{i=1}^{j-1} h_{i}^{2}\right)^{-1}\left(\prod_{i=1}^{r-2 t} b_{2 t+i}\right)^{-1} 1 \leq j \leq 11\right)
\end{aligned}
$$

induces an automorphism $\eta \in \operatorname{Aut}(H)$, then there exists a Riemann surface of genus (8) with a symmetry $\rho$, such that

$$
H_{\eta, 2}=H \rtimes\left\langle\rho: \rho^{2}=1\right\rangle \subseteq \operatorname{Aut}^{ \pm}(X) .
$$

Proof. Assume that a finite group $H$ has a generating vector $T_{r, \gamma}=\left[b_{1}, \ldots\right.$, $\left.b_{r}, h_{1}, \ldots, h_{\gamma}\right]$ which satisfies the condition (10). Then, the signature (6) with $m_{i}=\sharp\left(b_{i}\right)$ for $1 \leq i \leq r$ has the form

$$
\left(\gamma ; \pm ;\left[m_{1}, \ldots, m_{t}, m_{t}, \ldots, m_{1}, m_{2 t+1}, \ldots, m_{2 t+s}\right] ;\{-\}\right),
$$

where $s=r-2 t$ and the sign is - for $\gamma>0$ and it is + for $\gamma=0$. Let $y_{1}, \ldots, y_{a}$ with $a=\gamma+t$ be generating elliptic elements, and let $c_{0}, \ldots, c_{s}$ be generating reflections of an NEC group $\Lambda^{\prime}$ with the signature

$$
\left(0 ;+;\left[2, . ., 2, m_{1}, \ldots, m_{t}\right] ;\left\{\left(m_{2 t+1}, \ldots, m_{2 t+s}\right)\right\}\right) .
$$


Then, $\left(c_{j-1} c_{j}\right)^{m_{2 t+j}}=1$ for $1 \leq j \leq s, y_{i}^{2}=1$ for $1 \leq i \leq \gamma, y_{\gamma+i}^{m_{i}}=1$, for $1 \leq i \leq t$ and $\left(y_{1} \ldots y_{a}\right) c_{0}\left(y_{1} \ldots y_{a}\right)^{-1} c_{s}=1$. Let

$$
\begin{aligned}
d_{i} & =\left(y_{i} \ldots y_{a}\right) c_{0}\left(y_{i+1} \ldots y_{a}\right)^{-1} \quad \text { for } i=1, \ldots, \gamma \\
x_{j} & =y_{\gamma+j}, x_{t+j}=c_{0} y_{\gamma+t-j+1}^{-1} c_{0} \quad \text { for } j=1, \ldots, t \\
x_{2 t+j} & =c_{j-1} c_{j} \quad \text { for } j=1, \ldots s .
\end{aligned}
$$

Then, $d_{i} d_{i+1}=y_{i} y_{i+1}$ for $1 \leq i \leq \gamma-1$ what implies that

$$
\begin{aligned}
\prod_{i=1}^{\gamma} d_{i}^{2} & =d_{1} y_{1} y_{\gamma} d_{\gamma}=\left(\Pi_{j=1}^{a} y_{j}\right) c_{0}\left(\Pi_{j=1}^{a} y_{j}\right)^{-1}\left(\Pi_{j=1}^{t} y_{\gamma+j}\right) c_{0}\left(\Pi_{j=1}^{t} y_{\gamma+j}\right)^{-1} \\
& =c_{s}\left(\Pi_{j=1}^{t} y_{\gamma+j}\right) c_{0}\left(\Pi_{j=1}^{t} y_{\gamma+j}\right)^{-1} .
\end{aligned}
$$

Thus

$$
\prod_{j=1}^{2 t+s} x_{j}=\left(\prod_{j=1}^{t} y_{\gamma+j}\right) c_{0}\left(\prod_{j=1}^{t} y_{\gamma+j}\right)^{-1} c_{0}\left(c_{0} c_{s}\right)=\left(\prod_{i=1}^{\gamma} d_{i}^{2}\right)^{-1} .
$$

The elements $d_{1}, \ldots, d_{\gamma}, x_{1}, \ldots, x_{2 t+s}$ listed in (14) satisfy the relations

$$
\prod_{j=1}^{2 t+s} x_{j} \prod_{i=1}^{\gamma} d_{i}^{2}=1 \text { and } x_{i}^{m_{i}}=1 \quad \text { for } i=1, \ldots, r,
$$

and so, they generate a subgroup $\Lambda<\Lambda^{\prime}$ with the signature (12).

Let $\theta: \Lambda \rightarrow H$ be an epimorphism given by (9) and suppose that there exist a semidirect product of $H$ by a group $\mathbb{Z}_{2}=\left\langle\rho: \rho^{2}=1\right\rangle$ and a smooth epimorphism $\theta^{\prime}: \Lambda^{\prime} \rightarrow H^{\prime}=H \rtimes \mathbb{Z}_{2}$, such that $\rho=\theta^{\prime}\left(c_{0}\right)$ and $\left.\theta^{\prime}\right|_{H}=\theta$. Then, by (14), we have

$$
\theta^{\prime}\left(y_{\gamma+i}\right)=b_{i} \text { for } 1 \leq i \leq t
$$

and $\rho b_{i} \rho=b_{2 t-i+1}^{-1}$ and $\rho b_{t+i} \rho=b_{t-i+1}^{-1}$. Thus

$$
\begin{aligned}
h_{\gamma}=\theta^{\prime}\left(d_{\gamma}\right) & =\theta^{\prime}\left(y_{\gamma} \prod_{i=1}^{t} y_{\gamma+i} c_{0}\left(\prod_{i=1}^{t} y_{\gamma+i}\right)^{-1}\right)= \\
& =\theta^{\prime}\left(y_{\gamma}\right)\left(\prod_{i=1}^{t} b_{i}\right) \rho\left(\prod_{i=1}^{t} b_{i}\right)^{-1}= \\
& =\theta^{\prime}\left(y_{\gamma}\right) \prod_{i=1}^{2 t} b_{i} \rho=\theta^{\prime}\left(y_{\gamma}\right) B \rho \text { for } B=\prod_{i=1}^{2 t} b_{i} .
\end{aligned}
$$

Since $h_{\gamma}=\theta^{\prime}\left(y_{\gamma}\right) B \rho$ and $\rho B \rho=B^{-1}$, it follows that $\theta^{\prime}\left(y_{\gamma}\right)=h_{\gamma} B \rho$. The last element has order 2 what implies that $\rho h_{\gamma} \rho=B^{-1} h_{\gamma}^{-1} B$. Using the equalities $d_{i} d_{i+1}=y_{i} y_{i+1}$, we prove by induction that

$$
\theta^{\prime}\left(y_{i}\right)=h_{i} h_{i+1}^{2} \ldots h_{\gamma}^{2} B \rho \quad \text { for } i=1, \ldots, \gamma .
$$

These elements have orders 2 if and only if

$$
\begin{aligned}
\rho h_{i} \rho & =B^{-1}\left(h_{i+1}^{2} \ldots h_{\gamma}^{2}\right)^{-1} h_{i}^{-1}\left(h_{i+1}^{2} \ldots h_{\gamma}^{2}\right) B \\
& =C\left(h_{1}^{2} \ldots h_{i-1}^{2}\right) h_{i}^{-1}\left(h_{1}^{2} \ldots h_{i-1}^{2}\right)^{-1} C^{-1}
\end{aligned}
$$


for $C=\prod_{i=1}^{s} b_{2 t+i}$. Next, by the equality $x_{2 t+1}=c_{0} c_{1}$, we get $\theta^{\prime}\left(c_{1}\right)=$ $\rho b_{2 t+1}$. Thus, $\rho b_{2 t+1} \rho=b_{2 t+1}^{-1}$, because $\theta^{\prime}\left(c_{1}\right)$ has order 2 . Similarly by the equality $x_{2 t+2}=c_{1} c_{2}$, we get $\theta^{\prime}\left(c_{2}\right)=\rho b_{2 t+1} b_{2 t+2}$ what implies that $\rho b_{2 t+2} \rho=b_{2 t+1} b_{2 t+2}^{-1} b_{2 t+1}^{-1}$. In this way by induction, we prove that

$$
\theta^{\prime}\left(c_{j}\right)=\rho b_{2 t+1} \ldots b_{2 t+j} \quad \text { for } 1 \leq j \leq s
$$

and $\rho b_{2 t+j} \rho=\left(b_{2 t+1} \ldots b_{2 t+j-1}\right) b_{2 t+j}^{-1}\left(b_{2 t+1} \ldots b_{2 t+j-1}\right)^{-1}$.

Conversely, if a finite group $H$ has a generating vector $T_{r, \gamma}$ for which the assignment (11) induces an automorphism $\eta \in \operatorname{Aut}(H)$, then there is an action $\left(\Lambda^{\prime}, \theta^{\prime}, H_{\eta, 2}\right)$ on the Riemann surface $Y=\mathcal{H} / \operatorname{ker} \theta^{\prime}$, where $H_{\eta, 2}=H \rtimes$ $\left\langle\rho: \rho^{2}=1\right\rangle$ is the semidirect product with respect to the action $\rho h \rho=\eta(h)$ for $h \in H$, and $\Lambda^{\prime}$ is an NEC group with the signature (13), where $m_{i}=\sharp\left(b_{i}\right)$ for $1 \leq i \leq t$, and $\theta^{\prime}: \Lambda^{\prime} \rightarrow H_{\eta, 2}$ is a smooth epimorphism induced by (15), (16), (17) and $\theta^{\prime}\left(c_{0}\right)=\rho$. By the Hurwitz-Riemann formula, the surface $Y$ has genus $g$ given by (8) and it is symmetric, because $\rho$ is an anti-conformal involution.

We will say that a generating vector $T_{r, \gamma}=\left[b_{1}, \ldots, b_{r}, h_{1}, \ldots, h_{\gamma}\right]$ of a finite group $H$ is symmetric, if there is an integer $t$ in the range $1 \leq t \leq\left\lfloor\frac{r}{2}\right\rfloor$ for which the condition (10) is satisfied and the assignment (11) induces an automorphism $\eta \in \operatorname{Aut}(H)$. We will denote such a vector by $T_{r, \gamma}^{t, \eta}$.

Corollary 3.3. Any finite group acts on infinite many symmetric Riemann surfaces.

Proof. Clearly, this statement is true for the trivial group. Let $\left\{g_{1}, \ldots, g_{n}\right\}$ be a set of nonidentity generators of a finite nontrivial group $H$. By repeating one of elements $g_{i}$ two or three times if necessary, we can assume that $n \geq 3$. Then, $H$ has an unessential symmetric generating vector

$$
T_{2 n, 0}^{t, \eta}=\left[b_{1}, \ldots, b_{2 n}\right]=\left[g_{1}, \ldots, g_{t}, g_{t}^{-1} \ldots, g_{1}^{-1}\right] \quad \text { for } \eta=\operatorname{id}_{H} \text { and } t=n \text {, }
$$

and according to Theorem 3.2, there is a Riemann surface of genus $g=$ $1+|H|\left(-1+\sum_{i=1}^{t}\left(1-\frac{1}{\sharp\left(g_{i}\right)}\right)\right)$ with a symmetry $\rho$, such that

$$
H_{\eta, 2}=H \times\left\langle\rho: \rho^{2}=1\right\rangle \subseteq \operatorname{Aut}^{ \pm}(X) .
$$

The group $H$ acts on infinite many Riemann surfaces of different genera, because in the construction of generating vector, we can repeat generators of $H$ an infinite number of times.

Theorem 3.4. Let $A=\left\{a_{1}, \ldots, a_{n}\right\}$ be a minimal generating set of a finite noncyclic group $H$, and suppose that there is an automorphism $\varphi \in \operatorname{Aut}(H)$ of order 2, such that for each $a_{i} \in A, \varphi\left(a_{i}\right) \in\left\langle a_{j}\right\rangle$ for some $a_{j} \in A$. Then for any even $q \in \mathbb{N}$, there is a Riemann surface with an automorphism group $H \rtimes D_{q}$, where $D_{q}$ is a dihedral group generated by a conformal involution $v$ and a symmetry $\rho$, such that $\rho h \rho=h$ and $v h v=\varphi(h) \quad$ for $h \in H$.

Proof. Let $m_{i}=\sharp\left(a_{i}\right)$ for $i=1, \ldots, n$, and let $\alpha_{i j}$ be an integer, such that $\varphi\left(a_{i}\right)=a_{j}^{\alpha_{i j}}$ for $a_{i}, a_{j} \in A$ with $\varphi\left(a_{i}\right) \in\left\langle a_{j}\right\rangle$. If $j=i$, then $a_{i}=\varphi^{2}\left(a_{i}\right)=a_{i}^{\alpha_{i i}^{2}}$ 
what implies that $\alpha_{i i}^{2} \equiv 1\left(m_{i}\right)$. Thus, for $g_{i}=a_{i}$, we have $\varphi\left(g_{i}\right)=g_{i}^{-1}$ or $\varphi\left(g_{i}\right)=g_{i}$.

Now, assume that $i \neq j$. Since $\varphi\left(a_{i}\right)=a_{j}^{\alpha_{i j}}$, it follows that $m_{i}$ divides $m_{j}$. If $\varphi\left(a_{j}\right)=a_{k}^{\alpha_{j k}}$ for $k \neq i$, then $a_{i}=\varphi^{2}\left(a_{i}\right) \in\left\langle a_{k}\right\rangle$, against the assumption that $A$ is the minimal generating set. Thus, $\varphi\left(a_{j}\right)=a_{i}^{\alpha_{j i}}$ what implies that $m_{j}$ divides $m_{i}$. Consequently, $m_{i}=m_{j}$ and $\left(m_{i}, \alpha_{i j}\right)=1$. Since $a_{i}=\varphi^{2}\left(a_{i}\right)=$ $a_{i}^{\alpha_{i j} \alpha_{j i}}$, it follows that $\alpha_{i j} \alpha_{j i} \equiv 1\left(m_{i}\right)$. Thus, $\varphi\left(g_{i}\right)=g_{j}^{-1}$ and $\varphi\left(g_{j}\right)=g_{i}^{-1}$ for $g_{i}=a_{i}^{-\alpha_{j i}}$ and $g_{j}=a_{j}$.

Therefore, without lost of generality, we can assume that $H$ has a generating set $\left\{g_{1}, \ldots, g_{n}\right\}$, such that

$$
\begin{aligned}
\varphi\left(g_{2 i-1}\right) & =g_{2 i}^{-1}, \varphi\left(g_{2 i}\right)=g_{2 i-1}^{-1} \quad \text { for } i=1, \ldots, s, \\
\varphi\left(g_{2 s+i}\right) & =g_{2 s+i} \quad \text { for } i=1, \ldots, p, \\
\varphi\left(g_{2 s+p+i}\right) & =g_{2 s+p+i}^{-1} \quad \text { for } i=1, \ldots, u
\end{aligned}
$$

for some integers $s, p, u \geq 0$ with $2 s+p+u=n$.

For an even $q \in \mathbb{N}$, let $H^{\prime}$ be the semidirect product $H_{\varphi, q}=H \rtimes\left\langle\delta: \delta^{q}=\right.$ $1\rangle$ with respect to the action $\delta h \delta=\varphi(h)$ for $h \in H$. Then, for $\gamma=u+2-u_{(2)}$ and $r=2(s+p)$, there is a generating set $\left\{b_{1}, \ldots, b_{r}, h_{1}, \ldots, h_{\gamma}\right\}$ of $H^{\prime}$ which satisfies the conditions (7). It can be chosen as follows:

$$
\begin{aligned}
h_{u+1} & =\delta^{-1}, \quad h_{u+2}=\delta \text { if } u_{(2)}=0, \\
h_{i} & =\delta^{(-1)^{i+1}} g_{2 s+p+i}, \text { for } 1 \leq i \leq u \\
b_{i} & =g_{2 i}, \quad b_{s+2 p+i}=g_{2(s+1-i)}^{-1}, \quad \text { for } 1 \leq i \leq s . \\
b_{s+i} & =g_{2 s+i}, \quad b_{s+p+i}=g_{2 s+p+1-i}^{-1}, \quad \text { for } 1 \leq i \leq p .
\end{aligned}
$$

Any product of listed generators containing an odd number of $h_{i}$ has the form $h \delta^{l}$ for some $h \in H$ and an odd integer $l$. Therefore, it cannot be trivial, because otherwise $1 \neq \delta^{l} \in H$, a contradiction. Thus, $T_{r, \gamma}=\left[b_{1}, \ldots, b_{r}, h_{1}, \ldots, h_{\gamma}\right]$ is a generating vector of $H^{\prime}$, such that the condition (10) is satisfied for $t=s+p$. Let $\eta: H^{\prime} \rightarrow H^{\prime}$ be the mapping induced by (11). Then, $\eta\left(h_{i}\right)=h_{i}^{-1}$ for $1 \leq i \leq \gamma$. In particular, $\eta(\delta)=\delta^{-1}$ and

$$
g_{2 s+p+i}^{-1} \delta^{(-1)^{i}}=h_{i}^{-1}=\eta\left(h_{i}\right)=\eta\left(\delta^{(-1)^{i+1}}\right) \eta\left(g_{2 s+p+i}\right)=\delta^{(-1)^{i}} \eta\left(g_{2 s+p+i}\right)
$$

what implies $\eta\left(g_{2 s+p+i}\right)=\delta^{(-1)^{i+1}} g_{2 s+p+i}^{-1} \delta^{(-1)^{i}}=\varphi\left(g_{2 s+p+i}^{-1}\right)=g_{2 s+p+i}$ for $1 \leq i \leq u$.

Since $\eta\left(b_{i}\right)=b_{t+1-i}^{-1}$, it follows that $\eta\left(g_{2 s+i}\right)=g_{2 s+i}$ for $i=1, \ldots, p$ and $\eta\left(g_{2 i}\right)=g_{2 i}$ for $i=1, \ldots, s$. By the last equalities, we get

$$
\eta\left(g_{2 i-1}\right)=\eta\left(\varphi\left(g_{2 i}^{-1}\right)\right)=\eta\left(\delta g_{2 i}^{-1} \delta^{-1}\right)=\delta^{-1} g_{2 i}^{-1} \delta=\varphi^{q-1}\left(b_{2 i}^{-1}\right)=b_{2 i-1} .
$$

Summing up, $\left.\mu\right|_{H}=\mathrm{id}_{H}$ and $\eta(\delta)=\delta^{-1}$. Thus

$$
\eta\left(\delta g_{i} \delta^{-1}\right)=\delta^{-1} g_{i} \delta=\delta^{q-1} g_{i} \delta^{1-q}=\varphi^{q-1}\left(g_{i}\right)=\varphi\left(g_{i}\right)=\eta\left(\varphi\left(g_{i}\right)\right)
$$

what implies that $\eta\left(\delta g_{i} \delta^{-1}\right)=\eta\left(\varphi\left(g_{i}\right)\right)$ for $i=1, \ldots, n$. Therefore, $\eta$ is an automorphism of the group $H^{\prime}$ and vector $T_{r, \gamma}$ can be interpreted as a symmetric generating vector $T_{r, \gamma}^{s+p, \eta}$. According to Theorem 3.2, there is a 
Riemann surface with an automorphism group $H_{\eta, 2}^{\prime}=H^{\prime} \rtimes\langle\rho\rangle$ for a symmetry $\rho$ and the last group is isomorphic to $H \rtimes D_{q}$ for a dihedral group of order $2 q$ generated by conformal involution $v=\delta \rho$ and symmetry $\rho$.

Corollary 3.5. If an automorphism $\varphi \in A$ ut $(H)$ satisfies the assumptions of Theorem 3.4 and preserves a central element $z \in H$ of order 2 , then there is a symmetric Riemann surface $Y$ such that $H_{\varphi, 2}=H \rtimes\left\langle v: v^{2}=1\right\rangle \subseteq A u t^{+}(Y)$, where $v h v=\varphi(h)$ for $h \in H$.

Proof. For any central element $z \in Z(H)$ of order 2 , such that $\varphi(z)=z$, there is a normal subgroup $\left\langle\delta^{2} z^{-1}\right\rangle$ of $H_{\varphi, 4}=H \rtimes\left\langle\delta: \delta^{4}=1\right\rangle$. The quotient group $H_{\varphi / z}=H_{\varphi, 4} /\left\langle\delta^{2} z^{-1}\right\rangle$ has a symmetric generating vector $T_{r, \gamma}^{s+p, \eta}$ defined by (19). Thus, according to Theorem 3.2, there is a Riemann surface $Y$ with an automorphism group $G=H_{\varphi / z} \rtimes\langle\rho\rangle$, where $\rho$ is a symmetry, such that $\rho \delta \rho=\delta^{-1}$ and $\rho h \rho=h$ for $h \in H$. For $v=\delta \rho$, the subgroup $H \rtimes\langle v\rangle<G$ consists of all conformal automorphisms of $G$.

Using Theorems 3.2 and 3.4, we get the following examples of actions on symmetric Riemann surfaces:

Example 1. Let $H$ be a $(k, l, m)$-group generated by two elements $g_{1}$ and $g_{2}$ of orders $k$ and $l$, respectively, whose product has order $m$. There is an action $(\Lambda, \theta, H)$ on the Riemann surface $X=\mathcal{H} / \operatorname{ker} \theta$ for an NEC group $\Lambda$ with the signature $\sigma(\Lambda)=(0 ;+;[k, l, m] ;\{-\})$ and a smooth epimorphism $\theta: \Lambda \rightarrow H$ which maps $x_{1}$ and $x_{2}$ to $g_{1}$ and $g_{2}$, respectively. Singerman proved that if $H$ has an automorphism induced by the assignment

$$
\left(g_{1} \rightarrow g_{1}^{-1} \text { and } g_{2} \rightarrow g_{2}^{-1}\right) \text { or }\left(g_{1} \rightarrow g_{2}^{-1} \text { and } g_{2} \rightarrow g_{1}^{-1}\right),
$$

then $X$ is symmetric, where in the second case $k=l$. This result can be justified by applying Theorem 3.2 for an unessential generating vector

$$
T_{3,0}=\left[b_{1}, b_{2}, b_{3}\right]=\left[g_{1}, g_{2},\left(g_{1} g_{2}\right)^{-1}\right]
$$

of the group $H$. First, assume that there is $\varphi \in \operatorname{Aut}(H)$, such that $\varphi\left(g_{1}\right)=$ $g_{1}^{-1}$ and $\varphi\left(g_{2}\right)=g_{2}^{-1}$. Let $\eta=\varphi_{g_{1}} \circ \varphi$, where $\varphi_{g_{1}}$ is the conjugation via $g_{1}$ in $H$. Then

$$
\eta\left(b_{1}\right)=b_{1}^{-1}, \eta\left(b_{2}\right)=b_{1} b_{2}^{-1} b_{1}^{-1} \text { and } \eta\left(b_{3}\right)=\left(b_{1} b_{2}\right) b_{3}^{-1}\left(b_{1} b_{2}\right)^{-1} .
$$

Thus, vector (21) can be interpreted as a symmetric vector $T_{3,0}^{0, \eta}$, and by the proof of Theorem 3.2, there is an action $\left(\Lambda^{\prime}, \theta^{\prime}, H_{\eta, 2}\right)$ on symmetric Riemann surface $Y=\mathcal{H} / \operatorname{ker} \theta^{\prime}$, where $\Lambda^{\prime}$ is an NEC group with the signature $(0 ;+;[-] ;\{(k, l, m)\})$ and $\theta^{\prime}: \Lambda^{\prime} \rightarrow H_{\eta, 2}=H \rtimes\left\langle\rho: \rho^{2}=1\right\rangle$ is a smooth epimorphism given by $\theta^{\prime}\left(c_{0}\right)=\rho, \theta^{\prime}\left(c_{1}\right)=\rho g_{1}, \theta^{\prime}\left(c_{2}\right)=\rho g_{1} g_{2}$ and $\theta^{\prime}\left(c_{3}\right)=\rho$.

If $l=k$ and there is $\varphi \in \operatorname{Aut}(H)$ induced by the assignment $\varphi\left(g_{1}\right)=g_{2}^{-1}$ and $\varphi\left(g_{2}\right)=g_{1}^{-1}$, then vector $(21)$ can be interpreted as a symmetric vector $T_{3,0}^{t, \eta}$ for $t=1$ and $\eta=\varphi$. There is an action $\left(\Lambda^{\prime}, \theta^{\prime}, H_{\eta, 2}\right)$ on symmetric Riemann surface for an NEC group $\Lambda^{\prime}$ with the signature $(0 ;+;[k] ;\{(m)\})$ and a smooth epimorphism $\theta^{\prime}: \Lambda^{\prime} \rightarrow H_{\eta, 2}$ given by

$$
\theta^{\prime}\left(x_{1}\right)=g_{1}, \theta^{\prime}\left(c_{0}\right)=\rho, \theta^{\prime}\left(c_{1}\right)=\rho\left(g_{1} g_{2}\right)^{-1} .
$$


There are another actions of a $(k, l, m)$-group $H$ on symmetric Riemann surfaces which involve an automorphism $\varphi$ induced by the assignment (20). Let $H^{\prime}=H_{\varphi, q}=H \rtimes\left\langle\delta: \delta^{q}=1\right\rangle$ for an even $q \in \mathbb{N}$, and let $\eta$ be an automorphism of $H^{\prime}$ induced by $\eta(\delta)=\delta^{-1}$ and $\eta\left(g_{i}\right)=g_{i}$ for $i=1,2$. The group $H^{\prime}$ has a symmetric generating vector

$$
T_{0,4}^{0, \eta}=\left[\delta g_{1}, \delta^{-1} g_{2}, \delta^{-1}, \delta\right] \text { or } T_{2,2}^{1, \eta}=\left[g_{1}, g_{1}^{-1}, \delta^{-1}, \delta\right]
$$

according to whether $\varphi$ is induced by the first or by the second assignment in (20), respectively. According to Theorem 3.4, there is a symmetric Riemann surface with an automorphism group $H_{\eta, 2}^{\prime}=H^{\prime} \rtimes\left\langle\rho: \rho^{2}=1\right\rangle$ for a symmetry $\rho$. Vector $T_{0,4}^{0, \eta}$ corresponds to an action $\left(\Lambda^{\prime}, \theta^{\prime}, H_{\eta, 2}^{\prime}\right)$ on the Riemann surface of genus $g=1+q|H|$ for an NEC group $\Lambda^{\prime}$ with the signature $(0 ;+;[2,2,2,2] ;\{(-)\})$ and an epimorphism $\theta^{\prime}: \Lambda^{\prime} \rightarrow H_{\eta, 2}^{\prime}$ given by

$$
\theta^{\prime}\left(c_{0}\right)=\rho, \theta^{\prime}\left(x_{1}\right)=g_{1}^{-1} \delta^{-1} \rho, \theta^{\prime}\left(x_{2}\right)=\delta^{-1} g_{2} \rho, \theta^{\prime}\left(x_{3}\right)=\theta^{\prime}\left(x_{4}\right)=\delta \rho .
$$

Vector $T_{2,2}^{1, \eta}$ corresponds to the action $\left(\Lambda^{\prime}, \theta^{\prime}, H_{\eta, 2}^{\prime}\right)$ on the Riemann surface of genus $g=1+q|H|\left(1-\frac{1}{k}\right)$ for an NEC group $\Lambda^{\prime}$ with signature $(0 ;+;[2,2, k] ;\{(-)\})$ and $\theta^{\prime}$ induced by

$$
\theta^{\prime}\left(c_{0}\right)=\rho, \theta^{\prime}\left(x_{1}\right)=\theta^{\prime}\left(x_{2}\right)=\delta \rho \text { and } \theta^{\prime}\left(x_{3}\right)=g_{1} .
$$

Example 2. Let $H$ be the group generated by four elements $a, b, c, d$ which satisfy the relations

$$
\begin{aligned}
a^{4} & =1, \quad b^{4}=1, \quad c^{4}=1, \quad d^{4}=1, \\
{[a, b] } & =a^{2}, \quad[a, c]=a^{2} b^{2}, \quad[a, d]=b^{2}, \quad a^{2}=c^{2} \\
{[b, c] } & =a^{2}, \quad[b, d]=a^{2} b^{2}, \quad[c, d]=1, \quad b^{2}=d^{2} .
\end{aligned}
$$

It has order 64 . There are automorphisms $\varphi_{0}, \varphi_{1}, \varphi_{2}$ of $H$ induced by

$$
\begin{aligned}
& \varphi_{0}(a)=a^{-1}, \quad \varphi_{0}(b)=b^{-1}, \quad \varphi_{0}(c)=c^{-1}, \quad \varphi_{0}(d)=d^{-1}, \\
& \varphi_{1}(a)=a^{-1}, \quad \varphi_{1}(b)=b^{-1}, \quad \varphi_{1}(c)=c, \quad \varphi_{1}(d)=d, \\
& \varphi_{2}(a)=a, \quad \varphi_{2}(b)=b, \quad \varphi_{2}(c)=c^{-1}, \quad \varphi_{2}(d)=d^{-1} .
\end{aligned}
$$

(i) The group $H$ has unessential symmetric generating vectors

$$
T_{8,0}^{4, i d_{H}}=\left[a, b, c, d, d^{-1}, c^{-1}, b^{-1}, a^{-1}\right] \text { and } T_{8,0}^{2, \varphi_{2}}=\left[a, b, b^{-1}, a^{-1}, c, d, c^{-1}, d^{-1}\right]
$$

providing actions of $H$ on symmetric Riemann surfaces of genus $g=129$. Let $H_{\eta, 2}=H \rtimes\langle\rho\rangle$. According to Theorem 3.2, vector $T_{8,0}^{4, i d_{H}}$ corresponds to the action $\left(\Lambda^{\prime}, \theta^{\prime}, H_{\eta, 2}\right)$, where $\sigma\left(\Lambda^{\prime}\right)=(0 ;+;[4,4,4,4] ;\{(-)\})$ and $\theta^{\prime}$ is given by

$$
\theta^{\prime}\left(x_{1}\right)=a, \theta^{\prime}\left(x_{2}\right)=b, \theta^{\prime}\left(x_{3}\right)=c, \theta^{\prime}\left(x_{4}\right)=d \text { and } \theta^{\prime}\left(c_{0}\right)=\rho,
$$

and vector $T_{8,0}^{2, \varphi_{2}}$ corresponds to the action $\left(\Lambda^{\prime}, \theta^{\prime}, H_{\eta, 2}\right)$, where $\sigma\left(\Lambda^{\prime}\right)=(0 ;+[4,4] ;\{(4,4,4,4)\})$ and $\theta^{\prime}$ is given by

$$
\begin{aligned}
\theta^{\prime}\left(x_{1}\right)=a, \theta^{\prime}\left(x_{2}\right) & =b, \quad \theta^{\prime}\left(c_{0}\right)=\rho, \theta^{\prime}\left(c_{1}\right)=\rho c, \\
\theta^{\prime}\left(c_{2}\right)=\rho c d, \theta^{\prime}\left(c_{3}\right) & =\rho d, \quad \theta^{\prime}\left(c_{4}\right)=\rho .
\end{aligned}
$$


(ii) There is a normal subgroup $\left\langle\delta^{2} a^{2} b^{2}\right\rangle\left\langle H_{\varphi_{0}, 4}=H \rtimes\left\langle\delta: \delta^{4}=1\right\rangle\right.$ and the quotient group $H^{\prime}=H_{\varphi_{0}, 4} /\left\langle\delta^{2} a^{2} b^{2}\right\rangle$ has a symmetric essential generating vector

$$
T_{4,3}^{0, \eta}=\left[a, a, b, b, \delta c, \delta^{-1} d, \delta\right],
$$

for $\eta \in \operatorname{Aut}\left(H^{\prime}\right)$, such that $\left.\eta\right|_{H}=\varphi_{1}$ and $\eta(\delta)=\delta^{-1}$. Thus, there is an action $\left(\Lambda^{\prime}, \theta^{\prime}, H_{\eta, 2}^{\prime}\right)$ on a symmetric Riemann surface of genus $g=257$ for $H_{\eta, 2}^{\prime}=H^{\prime} \times\left\langle\rho: \rho^{2}=1\right\rangle$, where $\sigma\left(\Lambda^{\prime}\right)=(0 ;+;[2,2,2] ;\{(4,4,4,4)\})$ and $\theta^{\prime}$ given by

$$
\begin{aligned}
\theta^{\prime}\left(x_{1}\right) & =\delta c \rho, \theta^{\prime}\left(x_{2}\right)=\delta d \rho, \quad \theta^{\prime}\left(x_{3}\right)=\delta \rho, \theta^{\prime}\left(c_{0}\right)=\rho, \\
\theta^{\prime}\left(c_{1}\right) & =\rho a, \theta^{\prime}\left(c_{2}\right)=\rho a^{2}, \quad \theta^{\prime}\left(c_{3}\right)=\rho a^{2} b, \theta^{\prime}\left(c_{4}\right)=\rho a^{2} b^{2} .
\end{aligned}
$$

(iii) For an even integer $q \in \mathbb{N}$, the group $H^{\prime \prime}=G_{\varphi_{0}, q}=G \rtimes\left\langle\delta: \delta^{q}=1\right\rangle$ has a symmetric essential generating vector

$$
\left.T_{0,6}^{0, \eta}=\left[h_{1}, \ldots, h_{6}\right]=\delta a, \delta^{-1} b, \delta c, \delta^{-1} d, \delta^{-1}, \delta\right],
$$

where $\left.\eta\right|_{H}=$ id and $\eta(\delta)=\delta^{-1}$. There is an action $\left(\Lambda^{\prime}, \theta^{\prime}, H_{\eta, 2}^{\prime \prime}\right)$ on a symmetric Riemann surface of genus $g=1+128 q$, where $H_{\eta, 2}^{\prime \prime}=H^{\prime \prime} \rtimes$ $\langle\rho\rangle, \Lambda^{\prime}$ is an NEC group with the signature $(0 ;+;[2,2,2,2,2,2] ;\{(-)\})$ and $\theta^{\prime}: \Lambda^{\prime} \rightarrow H_{\eta, 2}^{\prime \prime}$ is an epimorphism given by $\theta^{\prime}\left(c_{0}\right)=\rho$ and

$$
\begin{aligned}
& \theta^{\prime}\left(x_{1}\right)=\delta^{-1} a \rho, \theta^{\prime}\left(x_{2}\right)=\delta^{-1} b \rho, \theta^{\prime}\left(x_{3}\right)=c^{-1} \delta^{-1} \rho, \\
& \theta^{\prime}\left(x_{4}\right)=\delta^{-1} d \rho, \quad \theta^{\prime}\left(x_{5}\right)=\delta \rho, \quad \theta^{\prime}\left(x_{6}\right)=\delta \rho .
\end{aligned}
$$

\section{On Asymmetric Generating Vectors}

A Riemann surface of genus $g \geq 2$ is called asymmetric or pseudo-real, if it has anti-conformal automorphisms, but none of them is a symmetry. The next result comes from [2]; however, its proof is different from the original.

Theorem 4.1. There is full action of a finite group $H$ on an asymmetric Riemann surface $X$ of genus $g \geq 2$ if and only if $H$ has an asymmetric generating vector by which we mean an essential exceptional generating vector $T_{r, \gamma}=$ $\left[b_{1}, \ldots, b_{r}, h_{1}, \ldots, h_{\gamma}\right]$ not being symmetric for $(\gamma, r)=(1,2),(3,0),(2,1)$. The genus $g$ of the surface $X$ is given by (8).

Proof. If $(\Lambda, \theta, H)$ is a full action on an asymmetric Riemann surface, then the group $\Lambda$ must have glide reflections but no reflection, and therefore, it has the signature

$$
\left(\gamma ;-;\left[m_{1}, \ldots, m_{r}\right] ;\{-\}\right) .
$$

A canonical generating set $\left\{d_{1}, \ldots, d_{\gamma}, x_{1}, \ldots, x_{r}\right\}$ of $\Lambda$ corresponds to an essential generating vector

$$
T_{r, \gamma}=\left[\theta\left(x_{1}\right), \ldots, \theta\left(x_{r}\right), \theta\left(d_{1}\right), \ldots, \theta\left(d_{\gamma}\right)\right]
$$

of $H$ which is exceptional, because $H$ acts on an asymmetric surface.

Conversely, if a finite group $H$ has an essential exceptional generating vector $T_{r, \gamma}=\left[b_{1}, \ldots, b_{r}, h_{1}, \ldots, h_{\gamma}\right]$, then the orders of all generators $h_{i}$ are 
divisible by 4 , and any composition of elements of vector $T_{r, \gamma}$ containing an odd number of $h_{i}$ neither is trivial nor has order 2. The signature (22) with $m_{i}=\sharp\left(b_{i}\right)$ for $1 \leq i \leq r$ appears on the lists of non-maximal signatures for $(\gamma, r)=(1,2),(3,0),(2,1)$, and it is maximal for all remaining pairs $(\gamma, r)$. There exists a maximal NEC group $\Lambda$ with any maximal signature. Let $\theta$ be an epimorphism induced by (9) from a maximal NEC group $\Lambda$ with the signature (22) onto $H$. Then, $\Gamma=\operatorname{ker} \theta$ is a surface Fuchsian group, and by the Hurwitz-Riemann formula, the genus of the Riemann surface $X=\mathcal{H} / \Gamma$ is equal to $(8)$.

Since $\Lambda$ is a maximal NEC group, it follows that $H \simeq \Lambda / \Gamma$ is the full automorphism group of $X$, because otherwise $\Lambda$ would be contained properly in another NEC group what contradicts the maximality of $\Lambda$. The surface $X$ has anti-conformal automorphisms $h_{i}$ and it has no symmetry, because no composition of elements of an exceptional vector containing an odd number of $h_{i}$ has order 2 . Therefore, $X$ is asymmetric.

A signature $(22)$ with $(\gamma, r) \in\{(1,2),(3,0),(2,1)\}$ is non-maximal and any NEC group $\Lambda$ with such a signature is a subgroup of another NEC group $\Lambda^{\prime}$. There are five such cases on the lists of non-maximal signatures

\begin{tabular}{rlrc} 
Case & \multicolumn{1}{l}{$\sigma(\Lambda)$} & $\sigma\left(\Lambda^{\prime}\right)$ & {$\left[\Lambda^{\prime}: \Lambda\right]$} \\
1 & $(2 ;-;[m] ;\{-\})$ & $(0 ;+;[2,2] ;\{(m)\})$ & 2 \\
2 & $(3 ;-;[-] ;\{-\})$ & $(0 ;+;[2,2,2] ;\{(-)\})$ & 2 \\
3 & $(1 ;-;[m, u] ;\{-\})$ & $(0 ;+;[2] ;\{(m, u)\})$ & 2 \\
4 & $(1 ;-;[m, m] ;\{-\})$ & $(0 ;+;[2, m] ;\{(-)\})$ & 2 \\
5 & $(1 ;-;[m, m] ;\{-\})$ & $(0 ;+;[-] ;\{(2,2,2, m)\})$ & 4.
\end{tabular}

All listed above pairs of signatures have been considered in the proof of Theorem 3.2. Indeed, in cases $(1)-(4)$, the signatures $\sigma(\Lambda)$ and $\sigma\left(\Lambda^{\prime}\right)$ have the forms (12) and (13), respectively. In case (5), the group $\Lambda^{\prime}$ contains a subgroup $\Lambda^{\prime \prime}$ with the signature $(0 ;+;[2, m] ;\{(-)\})$ which in turn contains a subgroup $\Lambda$ with the signature $(1 ;-;[m, m] ;\{-\})$. Now, $\sigma(\Lambda)$ and $\sigma\left(\Lambda^{\prime \prime}\right)$ have the forms (12) and (13), respectively. According to Theorem 3.2, if $H$ has a symmetric vector $T_{r, \gamma}$, then any action of this group with a nonmaximal signature $\sigma(\Lambda)$ on a Riemann surface $X$ extends to an action of the group $H \rtimes\langle\rho\rangle$ with the signature $\sigma\left(\Lambda^{\prime}\right)$ for some symmetry $\rho$, where in case (5), we should take $\sigma\left(\Lambda^{\prime \prime}\right)$ instead of $\sigma\left(\Lambda^{\prime}\right)$. It means that $H$ is not the full automorphism group and the surface $X$ is symmetric. Consequently, vector $T_{r, \gamma}$ cannot be symmetric for any action of $H$ with a non-maximal signature on an asymmetric Riemann surface.

Theorem 4.2. Let $A$ be a generating set of a finite group $H$, and let $\mu_{A^{\prime}}=$ $\operatorname{card}(A)-\sum_{h \in A^{\prime}} \frac{1}{\sharp(h)}$ for a subset $A^{\prime} \subseteq A$. Then

(i) for any $k \in \mathbb{N}$ and $\varphi \in \operatorname{Aut}(H)$ with $4 k_{(\sharp(\varphi))}=0$, there is an asymmetric Riemann surface $X$ of genus $g=1+4 k|H| \mu_{A^{\prime}}$, such that

$$
\operatorname{Aut}^{ \pm}(X) \simeq H_{\varphi, 4 k}=H \rtimes\left\langle\delta: \delta^{4 k}\right\rangle \text { and } \operatorname{Aut}^{+}(X) \simeq H \rtimes\left\langle\delta^{2}\right\rangle .
$$


(ii) if $\varphi^{2}=\mathrm{id}$ and $\varphi(z)=z$ for an element $z \in Z(H)$ of even order, such that $\varphi(h) h \neq z$ for all $h \in H$, then there is an asymmetric Riemann surface $X$ of genus $g=1+2|H| \mu_{A^{\prime}}$ with $\operatorname{Aut}^{ \pm}(X) \simeq H_{\varphi / z}$ and $\operatorname{Aut}^{+}(X) \simeq H$.

Proof. We will prove items $(i)$ and $(i i)$ simultaneously using symbol $H^{\prime}$ for $H_{\varphi, 4 k}$ and $H_{\varphi / z}$, respectively. Let us write the generating set of $H$ as a sum of two disjoint subsets $A=\left\{g_{k_{1}}, \ldots g_{k_{s}}\right\} \bigcup\left\{g_{l_{1}}, \ldots g_{l_{t}}\right\}$. Then, $H^{\prime}$ has a generating vector $T_{r, \gamma}=\left[b_{1}, \ldots, b_{r}, h_{1}, \ldots, h_{\gamma}\right]$ for $(\gamma, r)=(2 s+2,2 t)$, where

$$
\begin{aligned}
h_{2 s+1} & =\delta^{-1}, \quad h_{2 s+2}=\delta \\
h_{2 i-1} & =\delta g_{k_{i}}, \quad h_{2 i}=\left(\delta g_{k_{i}}\right)^{-1} \quad \text { for } 1 \leq i \leq s \\
b_{j} & =g_{l_{j}} b_{t+j}=g_{l_{t+1-j}}^{-1} \quad \text { for } 1 \leq j \leq t .
\end{aligned}
$$

To show that $T_{r, \gamma}$ is exceptional, we will prove first that $h_{1}, \ldots, h_{\gamma}$ have orders divisible by 4 . Let $\alpha_{i}=\sharp\left(\delta g_{i}\right)$ for $g_{i} \in A$. Then

$$
1=\left(\delta g_{i}\right)^{\alpha_{i}}=\varphi\left(g_{i}\right) \varphi^{2}\left(g_{i}\right) \ldots \varphi^{\alpha_{i}}\left(g_{i}\right) \delta^{\alpha_{i}},
$$

and so, $\delta^{\alpha_{i}} \in H$. In the group $H_{\varphi, 4 k}$, this is possible only when $\alpha_{i} \equiv 0(4 k)$. In $H_{\varphi / z}$, the element $\delta^{\alpha_{i}}$ belongs to $H$ only for even $\alpha_{i}$, because $\delta^{2}=z \in H$. Since $\varphi^{2}=\operatorname{id}_{H}$ and $z \in Z(H)$, it follows by (24) that:

$$
1=\left(\delta g_{i}\right)^{\alpha_{i}}=\left(\varphi\left(g_{i}\right) g_{i}\right)^{\frac{\alpha_{i}}{2}} \delta^{\alpha_{i}}=\left(\varphi\left(g_{i}\right) g_{i} z\right)^{\frac{\alpha_{i}}{2}},
$$

and so, $\sharp\left(\varphi\left(g_{i}\right) g_{i} z\right)$ divides $\frac{\alpha_{i}}{2}$. On the other hand, $\sharp\left(\varphi\left(g_{i}\right) g_{i} z\right)$ is divisible by $\sharp(z)$ which by assumption is even. Thus, $\alpha_{i} \equiv 0$ (4) also in the group $H_{\varphi / z}$.

Let $p$ be a composition of some generators listed in (23). If elements $h_{i}$ occur an odd number of times in $p$, then $p=h \delta^{\beta}$ for some $h \in H$ and an odd integer $\beta$. Thus, $p$ is nontrivial and $p^{2}=h \varphi^{\beta}(h) \delta^{2 \beta}$ for $2 \beta \not \equiv 0$ (4) what implies that $\sharp(p) \neq 2$ in the group $H_{\varphi, 4 k}$, because otherwise we get a contradiction that $1 \neq \delta^{2 \beta} \in H \bigcap\langle\delta\rangle$. In the group $H_{\varphi / z}$, we have $p=$ $h \delta^{\beta}=h z^{\frac{\beta-1}{2}} \delta=h^{\prime} \delta$ for $h^{\prime}=h z^{\frac{\beta-1}{2}} \in H$. Therefore, if $\sharp(p)=2$, then $h^{\prime} \varphi\left(h^{\prime}\right) \delta^{2}=1$ what implies $z=\delta^{2}=\left(h^{\prime} \varphi\left(h^{\prime}\right)\right)^{-1}=\varphi\left(h^{\prime-1}\right) h^{\prime-1}$. However, by assumption, $z$ cannot be written in this form. We have proved that the orders of generators $h_{1}, \ldots, h_{\gamma}$ are divisible by 4 and any composition of elements of vector $T_{\gamma, r}$ containing an odd number of $h_{i}$ neither is trivial nor has order 2 . Thus, $T_{\gamma, r}$ is an essential exceptional generating vector and $(\gamma, r)$ is different from $(1,2),(3,0)$ and $(2,1)$. According to Theorem 4.1, there is full action of $H^{\prime}$ on an asymmetric Riemann surface $X$ of genus $g=1+\left|H^{\prime}\right|\left(\operatorname{card}(A)-\sum_{j=1}^{t} \frac{1}{\sharp\left(g_{l_{j}}\right)}\right)$.

In case $(i)$, the subgroup $H \rtimes\left\langle\delta^{2}\right\rangle$ of $H_{\varphi, 4 k}$ has index 2 and all its generators are conformal because $\delta^{2}=h_{2 s+2}^{2}$ and any generator in $A$ is one of elements $b_{j}$ or $h_{2 s+1} h_{j}$. Thus, $H \rtimes\left\langle\delta^{2}\right\rangle=\mathrm{Aut}^{+}(X)$. By similar arguments, we get $H=\operatorname{Aut}^{+}(X)$ in $(i i)$.

For a finite nontrivial group $H$, let $(X, Y)_{g}^{H}$ denotes a pair of Riemann surfaces $X$ and $Y$ of the same genus $g \geq 2$ which have subgroups $H_{X} \subset \operatorname{Aut}^{ \pm}(X)$ and $H_{Y} \subset \operatorname{Aut}^{ \pm}(Y)$, such that $H_{X} \simeq H \simeq H_{Y}$. We say that $(X, Y)_{g}^{H}$ is an (a.s.)-pair, if $X$ is asymmetric and $Y$ is symmetric. A 
generating vector of $H$ for which there exists (a.s.)-pair $(X, Y)_{g}^{H}$ is called (a.s)-generating vector.

Theorem 4.3. Let $\mu=\sum_{j=1}^{n}\left(1-\frac{1}{\sharp\left(g_{j}\right)}\right)$ for a generating set $A=\left\{g_{1}, \ldots, g_{n}\right\}$ of a finite group $H$, and let $\varphi$ be an automorphism of $H$, such that $\varphi^{2}=\mathrm{id}_{H}$. Then, for any $k \in \mathbb{N}$, there is an (a.s.)-pair $(X, Y)_{g=1+4 k|H| \mu}^{H_{\varphi, 4 k}}$. Moreover, if $\varphi$ preserves a central element $z \in Z(H)$ of order 2 , then there is an (a.s.)-pair $(X, Y)_{g=1+2|H| \mu}^{H_{\varphi / z}}$.

Proof. Let $H^{\prime}=H_{\varphi, 4 k}=H \rtimes\left\langle\delta: \delta^{4 k}=1\right\rangle$ or let $H^{\prime}=H_{\varphi / z}=(H \rtimes\langle\delta$ : $\left.\left.\delta^{4}=1\right\rangle\right) /\left\langle\delta^{2} z\right\rangle$ on condition that there is a central element $z \in H$ of order 2 fixed by $\varphi$. Then, $H^{\prime}$ has an asymmetric generating vector

$$
T_{2 n, 2}=\left[g_{1}, \ldots, g_{n}, g_{n}^{-1}, \ldots, g_{1}^{-1}, \delta^{-1}, \delta\right] .
$$

Let $\eta: H^{\prime} \rightarrow H^{\prime}$ be the mapping, such that $\eta(\delta)=\delta^{-1}$ and $\eta(h)=h$ for $h \in$ $H$. Then, $\eta\left(\delta h \delta^{-1}\right)=\delta^{-1} h \delta=\delta^{4 k-1} h \delta^{1-4 k}=\varphi^{4 k-1}(h)=\varphi(h)=\eta(\varphi(h)$. Thus, $\eta$ is an automorphism of $H^{\prime}$ and vector $T_{2 n, 2}$ can be interpreted as a symmetric vector $T_{2 n, 2}^{n, \eta}$. According to Theorems 4.1 and 3.2, there is (a.s.)pair $(X, Y)_{g}^{H^{\prime}}$, where $g=1+4 k|H| \mu$ or $g=1+2|H| \mu$ according to whether $H^{\prime}=H_{\varphi, 4 k}$ or $H^{\prime}=H_{\varphi / z}$, respectively.

\section{On Fixed Point Free Generating Vectors}

We say that an action $(\Lambda, \theta, H)$ on the Riemann surface $X=\mathcal{H} / \operatorname{Ker} \theta$ is fixed point free, if none automorphism in $H$ has fixed points. In this chapter, we study fixed point free actions for which $\Lambda$ has a signature $\sigma_{\gamma}=(\gamma ;-;[-] ;\{-\})$ with $\gamma \geq 3$. Then, $H$ has a generating vector

$$
T_{0, \gamma}=\left[h_{1}, \ldots, h_{\gamma}\right]=\left[\theta\left(d_{1}\right), \ldots, \theta\left(d_{\gamma}\right)\right],
$$

where $d_{1}, \ldots, d_{\gamma}$ are canonical generating glide reflections of $\Lambda$. By Theorem 4.1, there is full fixed point free action of a finite group $H$ on an asymmetric Riemann surface $X$ if and only if $H$ has an exceptional generating vector $T_{0, \gamma}$ which is not symmetric for $\gamma=3$.

Theorem 5.1. Let $H$ be a finite group admitting a symmetric generating vector $T_{0, \gamma}^{0, \eta}=\left[h_{1}, \ldots, h_{\gamma}\right]$, where $\gamma>3$ and $\eta \in \operatorname{Aut}(H)$ is induced by the assignment

$$
h_{j} \rightarrow\left(h_{1}^{2} \ldots \cdot h_{j-1}^{2}\right) h_{j}^{-1}\left(h_{1}^{2} \ldots h_{j-1}^{2}\right)^{-1} \quad \text { for } 1 \leq j \leq \gamma .
$$

Then, there exists a pair $(X, Y)_{g}^{H}$ for $g=1+|H|(\gamma-2) / 2$, such that $Y / H$ is a hyperelliptic Klein surface of algebraic genus $\gamma-1$ Moreover, $(X, Y)_{g}^{H}$ is an (a.s.)-pair, if vector $T_{0, \gamma}^{0, \eta}$ is exceptional.

Proof. Assume that a finite group $H$ has a generating vector $T_{0, \gamma}=\left[h_{1}, \ldots, h_{\gamma}\right]$. If $\gamma>3$, then the signature $\sigma_{\gamma}$ is maximal and there is full action $\left(\Lambda_{\max }, \theta, H\right)$ on the Riemann surface $X=\mathcal{H} / \operatorname{ker} \theta$ of genus $g=1+|H|(\gamma-2) / 2$, where $\Lambda_{\max }$ is a maximal NEC group with the signature $\sigma_{\gamma}$ and $\theta$ is induced by 
$\theta\left(d_{i}\right)=h_{i}$ for $1 \leq i \leq \gamma$. If vector $T_{0, \gamma}$ is exceptional, then there is no symmetry in $H$ and $X$ is asymmetric. On the other hand, if the assignment (25) induces an automorphism $\eta \in \operatorname{Aut}(H)$, then there is an action $\left(\Lambda^{\prime}, \theta^{\prime}, H_{\eta, 2}\right)$ on a symmetric Riemann surface $Y$, where $\Lambda^{\prime}$ is an NEC group with the signature $(0 ;+;[2, . \gamma ., 2] ;\{(-)\})$ and $\theta^{\prime}: \Lambda^{\prime} \rightarrow H_{\eta, 2}=H \rtimes\left\langle\rho: \rho^{2}=1\right\rangle$ is given by

$$
\theta^{\prime}\left(c_{0}\right)=\rho \quad \text { and } \quad \theta^{\prime}\left(y_{i}\right)=h_{i} h_{i+1}^{2} \ldots h_{\gamma}^{2} \rho \quad \text { for } 1 \leq i \leq \gamma
$$

for a canonical generating reflection $c_{0}$ and canonical elliptic generators $y_{1}, \ldots, y_{\gamma}$ of $\Lambda^{\prime}$. The surface $Y$ is isomorphic to $\mathcal{H} / \Gamma$ for $\Gamma=\operatorname{ker} \theta^{\prime}$ and it has the same genus as $X$. The elements $\left(y_{i} \ldots y_{\gamma}\right) c_{0}\left(y_{i+1} \ldots y_{\gamma}\right)^{-1}$ for $i=1, \ldots, \gamma$ generate a subgroup $\Lambda<\Lambda^{\prime}$ of index 2 being an NEC group with the signature $\sigma_{\gamma}$ and $H \simeq \Lambda / \Gamma$. Thus, the orbit space $Z=Y / H \simeq \mathcal{H} / \Lambda$ is a Klein surface of algebraic genus $p=\gamma-1$ and

$$
\mathbb{Z}_{2} \simeq H_{\eta, 2} / H \simeq\left(\Lambda^{\prime} / \Gamma\right) /(\Lambda / \Gamma) \simeq \Lambda^{\prime} / \Lambda
$$

Since the algebraic genus of $\Lambda^{\prime}$ is equal to 0 , it follows that $\mathbb{Z}_{2}$ is generated by a hyperelliptic involution of the Klein surface $Z$.

Example 3. There is an infinite family of groups acting with the signature $\sigma_{3}$ on asymmetric Riemann surfaces. It was constructed by Marston Conder in [14]. Let $H$ be the 33-rd group of order 64 in the Small Groups Database. It is generated by two elements $g_{1}$ and $g_{2}$ satisfying the following relations:

$$
\left[g_{2}, g_{1}^{2}\right]=\left[g_{1},\left[g_{1}, g_{2}\right]\right],\left[\left[g_{1}, g_{2}\right], g_{1}^{2}\right]=g_{2}^{2} \text { and }\left[g_{1},\left[g_{1},\left[g_{1}, g_{2}\right]\right]\right]=g_{2}^{2} .
$$

Using MAGMA, it can be checked that

$$
T_{0,3}=\left[h_{1}, h_{2}, h_{3}\right]=\left[g_{1}, g_{1} \cdot\left[g_{1},\left[g_{1}, g_{2}\right]\right], g_{2}\right]
$$

is an exceptional generating vector of $H$ and the assignment (25) does not induce an automorphism of this group. Thus, by Theorem 4.1, there is an action $(\Lambda, \theta, H)$ on an asymmetric Riemann surface $X=\mathcal{H} / \operatorname{ker} \theta$ of genus 33 , where $\Lambda$ is an NEC group with the signature $\sigma_{3}$ and $\theta: \Lambda \rightarrow H$ is induced by $\theta\left(d_{i}\right)=h_{i}$ for $i=1,2,3$.

Let $\Gamma_{m}$ with $m \in \mathbb{N}$ be the characteristic subgroup of $\Gamma=\operatorname{ker} \theta$ generated by the commutator $[\Gamma, \Gamma]$ and all $m$ th powers of the generators of $\Gamma$ and their conjugates. Then, $\Gamma_{m}$ is also torsion-free and $\Gamma / \Gamma_{m}$ is an abelian group of order $m^{66}$ what implies that $\mu\left(\Gamma_{m}\right)=\mu(\Gamma) \cdot m^{66}$. The quotient group $G_{m}=\Lambda / \Gamma_{m}$ acts on the asymmetric Riemann surface $\mathcal{H} / \Gamma_{m}$ which by the Hurwitz-Riemann formula has genus $g=1+\left|G_{m}\right| / 2=1+\left|\mu\left(\Gamma_{m}\right) / \mu(\Lambda)\right| / 2=$ $32 m^{66}+1$.

Corollary 5.2. The lower sharp bound on the genus $g$ of an asymmetric Riemann surface with a fixed point free action of the automorphism group $H=$ $\operatorname{Aut}^{ \pm}(X)$ is equal to $1+|H|$ or $1+\frac{|H|}{2}$, according to whether $H$ is abelian or nonabelian.

Proof. An automorphism of an asymmetric Riemann surface $X$ of genus $g \geq$ 2 may have fixed points only when it is conformal. Thus, the group $H=$ 
$\operatorname{Aut}^{ \pm}(X)$ acts without fixed points on $X$ with the signature $\sigma_{\gamma}$ for $\gamma \geq 3$, and by the Hurwitz-Riemann formula, $X$ has genus $g=1+\frac{|H|}{2}(\gamma-2)$. According to Theorem 4.1, an action of $H$ with the signature $\sigma_{3}$ is possible on condition that the assignment (25) does not induce an automorphism of $H$. In particular, $H$ is not abelian. By Example 3, the lower sharp bound on $g$ in nonabelian case is equal to $g=1+\frac{|H|}{2}$. In abelian case, this bound is equal to $1+|H|$, because for any $g$ satisfying the congruence $g \equiv 1(4)$, the cyclic group $Z_{g-1}=\langle\delta\rangle$ has an exceptional generating vector $T_{0,4}=\left[\delta, \delta, \delta^{-1}, \delta^{-1}\right]$ providing an action of $Z_{g-1}$ with the signature $\sigma_{4}$ on an asymmetric Riemann surface of genus $g$.

Theorem 5.3. Let $G=\left\langle g_{1}, \ldots, g_{n}\right\rangle$ be a finite group which has an automorphism $\varphi_{0}$ induced by

$$
\varphi_{0}\left(g_{i}\right)=g_{i}^{-1} \text { for } 1 \leq i \leq n .
$$

Then, for any $k \in \mathbb{N}$ and $\varphi \in \operatorname{Aut}(G)$, such that $\left(\varphi \varphi_{0}\right)^{2}=1$ and $(4 k)_{(\sharp(\varphi))}=$ 0 , there is an (a.s.)-pair $(X, Y)_{1+4 k n|H|}^{G}$ for which $\operatorname{Aut}^{ \pm}(X) \simeq G_{\varphi, 4 k}=G \rtimes$ $\left\langle\delta: \delta^{4 k}=1\right\rangle$ and $\operatorname{Aut}^{ \pm}(Y) \supseteq G_{\varphi, 4 k} \rtimes\langle\rho\rangle$ for a symmetry $\rho$, where

$$
\rho g_{i} \rho=\varphi \circ \varphi_{0}\left(g_{i}\right) \text { and } \rho \delta \rho=\delta^{-1} \text {. }
$$

If $\varphi^{2}=1$ and $\varphi(z)=z$ for a central element $z \in Z(G)$ of order 2 , such that $h \varphi(h) \neq z$ for all $h \in G$, then there is an (a.s.)-pair $(X, Y)_{1+2 n|H|}^{G}$, such that $\operatorname{Aut}^{+}(X) \simeq G$ and $G \rtimes\langle v\rangle \subset \operatorname{Aut}^{+}(Y)$ for a conformal involution $v$, where $v g_{i} v=\varphi_{0}\left(g_{i}\right), 1 \leq i \leq n$.

Proof. Let $H_{1}$ denotes the semidirect product $G_{\varphi, 4 k}=G \rtimes\left\langle\delta: \delta^{4 k}=1\right\rangle$ with respect to the action $\delta g_{i} \delta^{-1}=\varphi\left(g_{i}\right)$ for $1 \leq i \leq n$. In the case when $\varphi^{2}=1$ and there is an element $z \in Z(G)$ of order 2 , such that $\varphi(z)=z$ and $h \varphi(h) \neq z$ for all $h \in G$, let $H_{2}$ be the group $G_{\varphi / z}=G_{\varphi, 4} /\left\langle\delta^{2} z\right\rangle$. The group $H_{j}$ for $j=1,2$ has an asymmetric generating vector

$$
T_{0,2 n+2}=\left[h_{1}, \ldots, h_{\gamma}\right]=\left[\delta g_{1},\left(\delta g_{1}\right)^{-1}, \ldots, \delta g_{n},\left(\delta g_{n}\right)^{-1}, \delta^{-1}, \delta\right],
$$

such that $\eta\left(h_{i}\right)=h_{i}^{-1}$ for $1 \leq i \leq \gamma=2 n+2$, where $\eta: H_{j} \rightarrow H_{j}$ is the homomorphism induced by $(25)$. We have $\eta(\delta)=\delta^{-1}$ and

$$
g_{i}^{-1} \delta^{-1}=h_{2 i-1}^{-1}=\eta\left(h_{2 i-1}\right)=\eta(\delta) \eta\left(g_{i}\right)=\delta^{-1} \eta\left(g_{i}\right)
$$

what implies that $\eta\left(g_{i}\right)=\delta g_{i}^{-1} \delta^{-1}=\varphi \circ \varphi_{0}\left(g_{i}\right)$ for $1 \leq i \leq n$ and so $\left.\eta\right|_{H}=\varphi \circ \varphi_{0}$. Since by the assumptions $\left(\varphi \circ \varphi_{0}\right)^{2}=\operatorname{id}_{H}$, it follows that $\left.\eta\right|_{H} \circ \varphi=\varphi_{0}$. Thus

$$
\eta\left(\varphi\left(g_{i}\right)\right)=\varphi_{0}\left(g_{i}\right)=\delta^{-1} \eta\left(g_{i}\right) \delta=\eta(\delta) \eta\left(g_{i}\right) \eta(\delta)^{-1}=\eta\left(\delta g_{i} \delta^{-1}\right) .
$$

Therefore, $\eta$ is an automorphism of the group $H_{\varphi, 4 k}$ and vector (28) is not only an asymmetric generating vector, but it can be also interpreted as a symmetric generating vector $T_{0, \gamma}^{0, \eta}$. According to Theorems 4.2 and 3.2, there is an (a.s.)-pair $(X, Y)_{g}^{H_{j}}$ with $g=1+n\left|H_{j}\right|$, such that $\operatorname{Aut}^{ \pm}(X)=H_{j}$ and $\operatorname{Aut}^{ \pm}(Y) \supseteq H_{j} \rtimes\left\langle\rho: \rho^{2}=1\right\rangle$, where $\rho g_{i} \rho=\varphi \circ \varphi_{0}\left(g_{i}\right)$ and $\rho \delta \rho=\delta^{-1}$. The element $v=\delta \rho \in H_{j}$ is a conformal involution and $G \subset H_{j}$ is a subgroup 
generated by conformal automorphisms. Thus, for $j=2, \mathrm{Aut}^{+}(X)=G$ and $\operatorname{Aut}^{+}\left(Y_{2}\right) \supseteq G \rtimes\langle v\rangle$, where $v g_{i} v=g_{i}^{-1}$ for $1 \leq i \leq n$.

Corollary 5.4. For any finite noncyclic group $G=\left\langle g_{1}, \ldots, g_{m}\right\rangle$ which has an automorphism $\varphi_{0} \in \operatorname{Aut}(G)$ induced by (26) and for any $k \in \mathbb{N}$, there is an (a.s.)-pair $(X, Y)^{G_{\varphi_{0}, k}}$, such that

$$
\operatorname{Aut}^{ \pm}(X)=G_{\varphi_{0}, k} \text { and } \operatorname{Aut}^{ \pm}(Y) \supseteq G_{\varphi_{0}, k} \rtimes\left\langle\rho: \rho^{2}=1\right\rangle,
$$

where $\rho g_{i} \rho=g_{i}$ and $\rho \delta \rho=\delta^{-1}$.

Proof. This is the particular case of Theorem 5.3 for $\varphi=\varphi_{0}$.

Theorem 5.5. Let $p=\frac{g-1}{n}\left(\frac{n}{2}-1\right)$ for $n, g \in \mathbb{N}$, such that $g \geq 3$ is odd and $n$ is an even divisor of $g-1$. Then, there exists a symmetric p-hyperelliptic Riemann surface of genus $g$ being $n$-sheeted unbranched covering of a hyperelliptic Klein surface of algebraic genus $\frac{2(g-1)}{n}+1$. In particular, for any odd $g \geq 3$, there exists a symmetric hyperelliptic Riemann surface of genus $g$ with two commuting symmetries.

Proof. Let $\Lambda^{\prime}$ be an NEC group with the signature

$$
(0 ;+;[2, .2 t, 2] ;\{(-)\}) \text { for } t=\frac{g-1}{n}+1,
$$

and let $H^{\prime}$ be a semidirect product $\left\langle\delta: \delta^{n}=1\right\rangle \rtimes\left\langle\rho: \rho^{2}=1\right\rangle$ with respect to the action $\rho \delta \rho=\delta^{-1}$. Then, there is a smooth epimorphism $\theta^{\prime}: \Lambda^{\prime} \rightarrow H^{\prime}$ induced by

$$
\theta\left(c_{0}\right)=\rho \text { and } \theta\left(y_{1}\right)=\cdots=\theta\left(y_{2 t}\right)=\delta \rho,
$$

where $c_{0}$ is the canonical reflection and $y_{1}, \ldots, y_{2 t}$ are canonical elliptic elements of the group $\Lambda^{\prime}$. Applying the Hurwitz-Riemann formula for $\Lambda^{\prime}$ and $\Gamma=\operatorname{ker} \theta^{\prime}$, we calculate that the Riemann surface $Y=\mathcal{H} / \Gamma$ has genus $g$. Clearly, $Y$ is symmetric, because $\rho$ is an anti-conformal involution. The group $\Lambda^{\prime}$ has a subgroup $\Lambda$ of index 2 with the signature $\sigma_{2 t}=(2 t ;-;[-] ;\{-\})$ generated by glide reflections $d_{i}=\left(y_{i} \ldots y_{2 t}\right) c_{0}\left(y_{i+1} \ldots y_{2 t}\right)^{-1}$ for $i=1, \ldots, 2 t$. Let $\theta=\left.\theta^{\prime}\right|_{\Lambda}$. Then, $\theta: \Lambda \rightarrow \mathbb{Z}_{n}$ is a smooth epimorphism and $H=\mathbb{Z}_{n}$ has a symmetric generating vector $T_{0,2 t}^{0, \eta}=\left[\theta\left(d_{1}\right), \ldots, \theta\left(d_{2 t}\right)\right]=\left[\delta, \delta^{-1}, . t ., \delta, \delta^{-1}\right]$, where $\eta(\delta)=\delta^{-1}$. Thus, by Theorem 5.1 , there exists a pair $(X, Y)_{g}^{\mathbb{Z}_{n}}$, such that $Y / H$ is the Klein surface $\mathcal{H} / \Lambda$ of algebraic genus $2 t-1=\frac{2(g-1)}{n}+1$.

The subgroup $G<H^{\prime}$ generated by $\delta^{2}$ and $v=\delta \rho$ has index 2 and it consists of all conformal automorphisms in $H^{\prime}$. Thus, $G=\Lambda^{\prime+} / \Gamma$ for the canonical Fuchsian subgroup $\Lambda^{\prime+}<\Lambda^{\prime}$ which by (4), has the signature $(0 ;+;[2, .4 t ., 2] ;\{-\})$. The elements $x_{i}=y_{i}$ and $x_{2 t+i}=c_{0} y_{2 t+1-i} c_{0}$ for $1 \leq i \leq 2 t$ can be taken as the canonical generators of $\Lambda^{\prime+}$. Let $\mu=\left.\theta^{\prime}\right|_{\Lambda^{\prime+}}$. Then, $\mu\left(x_{i}\right)=v$ and $\mu\left(x_{2 t+i}\right)=\rho v \rho=\delta^{-2} v$ for $1 \leq i \leq 2 t$. Any element $h \in G$ has the form $h=\delta^{s} v^{r}$ for an even $s$ and $r=1$ or 0 . Thus, $h\left(\delta^{-2} v\right) h^{-1}=v$ if and only if $\delta^{2 s \pm 2}=1$. By the last equality, $\frac{n}{2}$ divides an odd integer $s \pm 1$ which is possible only when $n \equiv 2$ (4). Thus, there are $4 t$ or $2 t$ elements $\mu\left(x_{i}\right)$ which are conjugate with $v$ in $G$, according to whether $n \equiv 2(4)$ or $n \equiv 0(4)$, respectively. Moreover, $N_{G}(\langle v\rangle)=\{1, v\}$ 
in the first case and $\left.N_{G}\langle v\rangle\right)=\left\{1, v, \delta^{\frac{n}{2}}, v \delta^{\frac{n}{2}}\right\}$ in the second one. Thus, by Theorem 2.1, $v$ fixes $4 t$ points on $X$, and so, $\mathbb{Z}_{2}=\langle v\rangle=\Delta / \Gamma$ for an NEC group $\Delta$ with the signature $(p ;+;[2, . .4, .2] ;\{-\})$, where $p$ denotes the genus of the orbifold $X /\langle v\rangle$. Applying the Hurwitz-Riemann formula for $(\Delta, \Gamma)$, we get $p=\frac{g+1}{2}-t=(t-1)\left(\frac{n}{2}-1\right)=\frac{g-1}{n}\left(\frac{n}{2}-1\right)$. In particular, $p=0$ for $n=2$, and so, there exists a symmetric Riemann surface $Y$ with two commuting symmetries $\delta$ and $\rho$, such that $\delta \rho$ is the hyperelliptic involution.

Corollary 5.6. For any $p, d \in \mathbb{N}$, such that $d$ divides $p$, there exists a symmetric p-hyperelliptic Riemann surface of genus $g=\frac{2 p(d+1)}{d}+1$ being $2(d+1)$ sheeted unbranched covering of a hyperelliptic Klein surface of algebraic genus $\frac{2 p}{d}+1$. In particular, for any $p \geq 1$, there exists a symmetric $p$-hyperelliptic Riemann surface of genus $g=4 p+1$ being 4-sheeted unbranched covering of a hyperelliptic Klein surface of algebraic genus $2 p+1$.

Proof. This is a particular case of Theorem 5.5 for $n=2(d+1)$ and $t=\frac{p}{d}+1$.

\section{Acknowledgements}

We thank the referee for useful comments and suggestions.

Open Access. This article is licensed under a Creative Commons Attribution 4.0 International License, which permits use, sharing, adaptation, distribution and reproduction in any medium or format, as long as you give appropriate credit to the original author(s) and the source, provide a link to the Creative Commons licence, and indicate if changes were made. The images or other third party material in this article are included in the article's Creative Commons licence, unless indicated otherwise in a credit line to the material. If material is not included in the article's Creative Commons licence and your intended use is not permitted by statutory regulation or exceeds the permitted use, you will need to obtain permission directly from the copyright holder. To view a copy of this licence, visit http:// creativecommons.org/licenses/by/4.0/.

Publisher's Note Springer Nature remains neutral with regard to jurisdictional claims in published maps and institutional affiliations.

\section{References}

[1] Alling, N.L., Greenleaf, N.: Foundations of the theory of Klein surfaces, Lecture Notes in Mathematics, vol. 219. Springer, Heidelberg (1971)

[2] Bagiński, C., Gromadzki, G.: Minimal genus for pseudo-real Riemann surfaces. Arch. Math. 95, 481-492 (2010)

[3] Bujalance, E., Conder, M.: On cyclic groups of automorphisms of Riemann surfaces. J. Lond. Math. Soc. 59(2), 573-584 (1999)

[4] Bujalance, E., Costa, A.F.: Automorphism groups of pseudo-real Riemann surfaces of low genus. Acta Math. Sin. (Engl. Ser.) 30(1), 11-22 (2014)

[5] Bujalance, E., Conder, M.D.E., Costa, A.F.: Pseudo-real Riemann surfaces and chiral regular maps. Trans. AMS 362(7), 3365-3376 (2010) 
[6] Bujalance, E., Turbek, P.: Asymmetric and pseudo-symmetric hyperelliptic surfaces. Manuscr. Math. 108, 1-11 (2002)

[7] Earle, C.J.: On the moduli of closed Riemann surfaces with symmetries, advances in the theory of Riemann surfaces. Ann. Math. Stud. 66, 119-130 (1971)

[8] Estévez, J.L., Izquierdo, M.: Non-normal pairs of non-Euclidean crystallographic groups. Bull. Lond. Math. Soc. 38, 113-123 (2006)

[9] Etayo Gordejuela, J.J.: Nonorientable automorphisms of Riemann surfaces. Arch. Math. (Basel) 45(4), 374-384 (1985)

[10] Hidalgo, R.A.: Non-hyperelliptic Riemann surfaces with real field of moduli but not definable over the reals. Arch. Math. 93, 219-224 (2009)

[11] Hidalgo, R.A.: Erratum to: Non-hyperelliptic Riemann surfaces with real field of moduli but not definable over the reals. Arch. Math. 98, 449-451 (2012)

[12] Kozłowska-Walania, E., Tyszkowska, E.: On asymmetric $p$-hyperelliptic Riemann surfaces. Ann. Acad. Sci. Fenn. Math. 40, 669-682 (2015)

[13] Kozłowska-Walania, E., Tyszkowska, E.: On a certain family of asymmetric Riemann surfaces with the cyclic automorphism group. Kodai Math. J. 39(3), 510-520 (2016)

[14] Kozłowska-Walania, E., Tyszkowska, E.: Fixed points on asymmetric Riemann surfaces. Mediterr. J. Math 17, 131 (2020). https://doi.org/10.1007/ s00009-020-01565-91660-5446/20/040001-22

[15] Macbeath, A.M.: The classification of non-Euclidean crystallographic groups. Can. J. Math. 19, 1192-1205 (1967)

[16] Macbeath, A.M.: Action of automorphisms of a compact Riemann surface on the first homology group. Bull. Lond. Math. Soc. 5, 103-108 (1973)

[17] Preston, R.: Projective structures and fundamental domains on compact Klein surfaces, Ph.D. thesis University of Texas (1975)

[18] Singerman, D.: On the structure of non-Euclidean crystallographic groups. Proc. Camb. Philos. Soc. 76, 233-240 (1974)

[19] Singerman, D.: Symmetries and pseudo-symmetries of hyperelliptic surfaces. Glasg. Math. J. 21(1), 39-49 (1980)

[20] Singerman, D.: The remarkable Accola-Maclachlan surfaces. Contemp. Math. 629, 315-322 (2014) [Special Issue: Riemann and Klein Surfaces, Automorphisms, Symmetries and Moduli Spaces]

[21] Singerman, D.: Symmetries of Riemann surfaces with large automorphism groups. Math. Ann. 210, 17-32 (1974)

[22] Seppälä, M.: Complex algebraic curves with real moduli. J. Reine Angew. Math. 387, 209-220 (1988)

[23] Shimura, G.: On the field or rationality for an Abelian variety. Nagoya Math. J. 45, 167-178 (1971)

[24] Tyszkowska, E.: On (q,n)-gonal pseudo-real Riemann surfaces, Int. J. Math. 28(13), 1750095 (2017)

[25] Wilkie, H.C.: On non-Euclidean crystallographic groups. Math. Z. 97, 87-102 (1966) 
Ewa Tyszkowska

Institute of Mathematics

Gdańsk University

Wita Stwosza 57

80-952 Gdańsk

Poland

e-mail: ewa.tyszkowska@mat.ug.edu.pl

Received: July 22, 2020.

Revised: July 8, 2021.

Accepted: July 18, 2021. 